\title{
Burden of Sleep Disturbance During COVID-19 Pandemic: A Systematic Review
}

\author{
Ying Ni Lin ${ }^{1,2, *}$ \\ Zhuo Ran Liu (D) ${ }^{3, *}$ \\ Shi Qi Li (iD) ${ }^{1,2, *}$ \\ Chuan Xiang $\mathrm{Li}^{1,2,4}$ \\ Liu Zhang $\mathbb{( D}^{1,2}$ \\ Ning $\mathrm{Li}^{1,2}$ \\ Xian Wen Sun ${ }^{1,2}$ \\ Hong Peng $\mathrm{Li}^{1,2}$ \\ Jian Ping Zhou ${ }^{1,2}$ \\ Qing Yun $\mathrm{Li}^{1,2}$
}

'Department of Respiratory and Critical Care Medicine, Ruijin Hospital, Shanghai Jiao Tong University School of Medicine, Shanghai, 200025, People's Republic of China; ${ }^{2}$ Institute of Respiratory Disease, Shanghai Jiao Tong University School of Medicine, Shanghai, 200025, People's Republic of China; ${ }^{3}$ Department of Thyroid and Vascular Surgery, Ruijin Hospital, Shanghai Jiao Tong University School of Medicine, Shanghai, 200025, People's Republic of China; ${ }^{4}$ Department of Respiratory Medicine, Wuhan No.3 Hospital, Wuhan, 430000, People's Republic of China

*These authors contributed equally to this work
Correspondence: Qing Yun Li

Department of Respiratory and Critical Care Medicine, Ruijin Hospital, Shanghai Jiao Tong University School of Medicine, Shanghai, 200025, People's Republic of China

Tel +8I-2I-64370045

Email liqingyun68@hotmail.com

\begin{abstract}
Coronavirus disease 2019 (COVID-19) pandemic may exert adverse impacts on sleep among populations, which may raise awareness of the burden of sleep disturbance, and the demand of intervention strategies for different populations. We aimed to summarize the current evidence for the impacts of COVID-19 on sleep in patients with COVID-19, healthcare workers (HWs), and the general population. We searched PubMed and Embase for studies on the prevalence of sleep disturbance. Totally, 86 studies were included in the review, including 16 studies for COVID-19 patients, 34 studies for HWs, and 36 studies for the general population. The prevalence of sleep disturbance was $33.3 \%-84.7 \%$, and $29.5-$ $40 \%$ in hospitalized COVID-19 patients and discharged COVID-19 survivors, respectively. Physiologic and psychological traumatic effects of the infection may interact with environmental factors to increase the risk of sleep disturbance in COVID-19 patients. The prevalence of sleep disturbance was $18.4-84.7 \%$ in HWs, and the contributors mainly included high workloads and shift work, occupation-related factors, and psychological factors. The prevalence of sleep disturbance was $17.65-81 \%$ in the general population. Physiologic and social-psychological factors contributed to sleep disturbance of the general population during COVID-19 pandemic. In summary, the sleep disturbance was highly prevalent during COVID-19 pandemic. Specific health strategies should be implemented to tackle sleep disturbance.
\end{abstract}

Keywords: sleep disturbance, COVID-19 pandemic, SARS-CoV2

\section{Introduction}

Coronavirus disease 2019 (COVID-19) is an infectious disease caused by severe acute respiratory syndrome coronavirus 2 (SARS-CoV-2). COVID-19 outbreak, declared as a global pandemic by the World Health Organization (WHO) on March 11th 2020, has presented an unprecedented challenge to public health systems and caused global economic crises. The uncertainties and fears towards COVID-19, along with the societal consequences of mass lockdown, may lead to sleep disturbance and psychological burdens on a large number of individuals, including patients with COVID-19, healthcare workers (HWs), and the general public.

Sleep plays an essential role on regulation of psychological and physical processes. ${ }^{1}$ Poor sleep and sleep disturbance could interact with psychological and physical disorders to worsen health consequences among populations. Several studies have reported impacts of COVID-19 on sleep in specific populations. ${ }^{2-4}$ Sleep disorders may exert negative impacts on the process, prognosis, and rehabilitation of patients with COVID-19. Sleep disorders also affect the working ability 
of HWs. COVID-19-associated societal responses including home confinement, school suspension, and social isolation also increase the likelihood of sleep disturbance in the general public. However, risk factors for sleep disturbance and its associated health consequences still need to be addressed. Thus, we summarized the current evidence on the prevalence and associated factors of sleep disturbance in patients with COVID-19, HWs, and the general public. The increasing evidence addresses the necessity of awareness and interventions of sleep disturbance during and after COVID-19 pandemic.

\section{Methods}

\section{Search Strategy}

Electronic searches were performed in PubMed and Embase, and were updated on Dec 10th, 2020. The following terms were used for the searches, ie (sleep) OR (sleep disturbance) OR (sleep disorders) OR (sleep problems) OR (insomnia) OR (sleep apnea) OR (sleep breathing disorders) AND (COVID-19) OR (SARS-CoV2). The reference lists of full articles were also searched for relevant publications. The searches were conducted, and the full-text articles were reviewed and analyzed by 2 independent researchers (Lin $\mathrm{YN}$ and $\mathrm{Li} \mathrm{SQ}$ ). In case of disagreement between the two reviewers, a third reviewer (Liu ZR) reviewed the articles and consensus among the three reviewers was reached.

\section{Study Selection}

Studies were included if (1) the studies were cross-sectional, longitudinal, prospective, retrospective, or case-series in design; (2) the studies targeted populations including HWs, the general public, and COVID-19 patients; (3) the studies provided data of prevalence and/or risk factors of sleep disturbance; (4) the studies were written in English. Studies were excluded if (1) the full-text were unavailable; (2) studies were not written in English; (3) they were reviews, meta-analysis, conference abstracts, and protocols.

We initially identified 1430 studies. After removing 498 duplicates, we screened the remaining 932 studies by reviewing the titles and abstracts. Totally 136 studies were assessed for eligibility, and finally 86 studies were included in the review (Figure 1).

\section{Assessment of Study Quality}

Study quality was assessed using the Loney criteria through eight items including study design and sampling

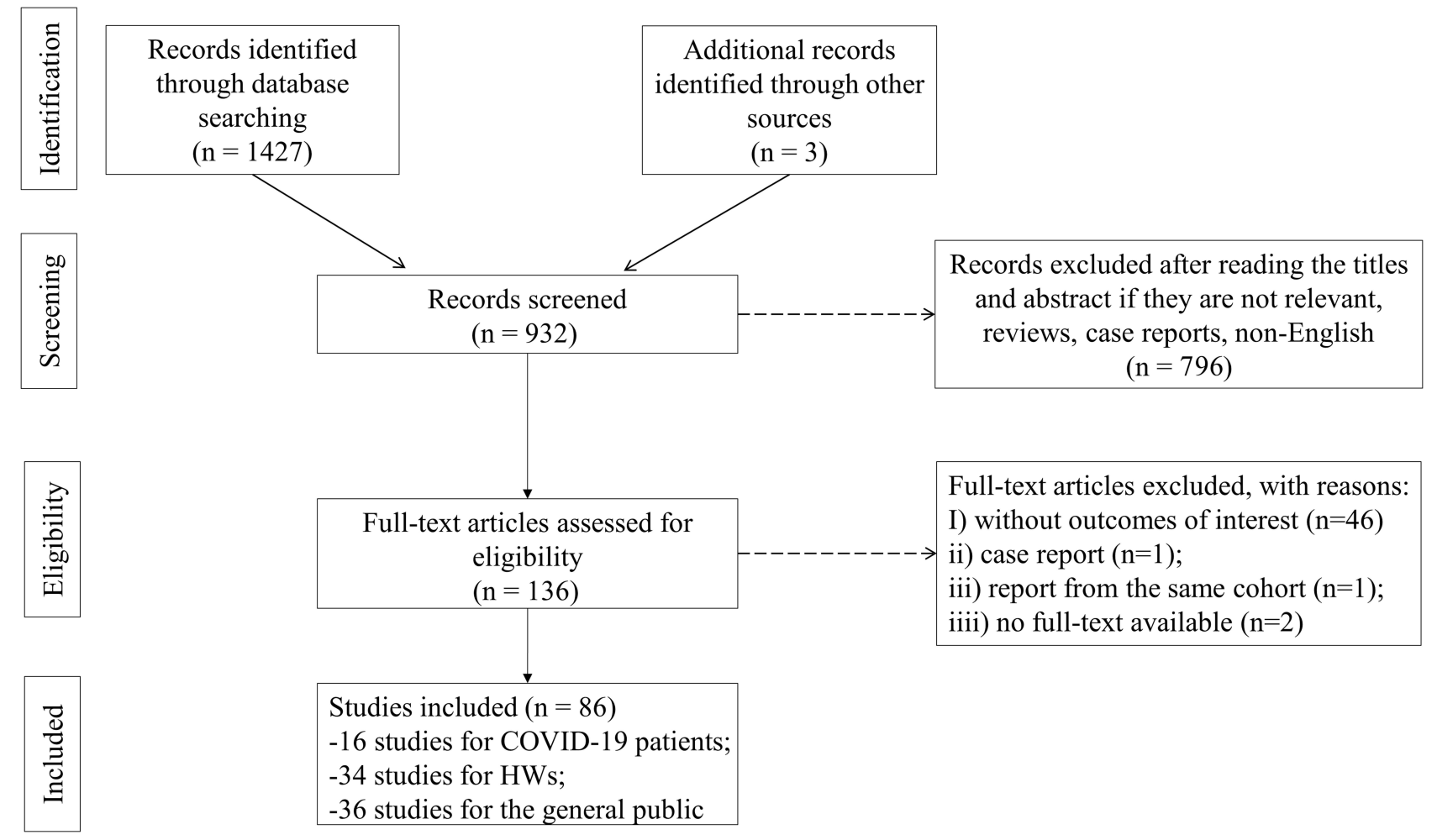

Figure I Flowchart of literature selection. 
method, unbias sampling frame, sample size, appropriate measurement, unbiased measurement, response rate, estimates of prevalence, and description of study subjects. ${ }^{5}$ Scores range from 0 to 8 points. A total score of $7-8$ is considered as high quality, 4-6 as moderate quality, and 0-3 as low quality. The detailed quality assessment of the studies was shown in Table S1. Study quality was assessed independently by two researchers (Lin YN and Li SQ). In case of disagreement, a third reviewer (Liu ZR) reassessed the studies and consensus among the three reviewers was reached.

\section{Results and Discussion Sleep Disturbance in Patients with COVID-19 \\ Hospitalized Patients}

The prevalence of sleep disturbance (ranging from 33.3\% to $84.7 \%$ ) in hospitalized COVID-19 patients was reported in 6 studies $^{6-11}$ (Table 1). A retrospective study reviewed the psychiatric medical records of 329 COVID-19 patients, and showed $25.5 \%$ received psychiatric consultations, $33 \%$ of whom were diagnosed with sleep disorders (insomnia, early awakening, difficulty falling asleep), and $22.6 \%$ and $54.8 \%$ were prescribed benzodiazepines and non-benzodiazepine sedative-hypnotics (zolpidem), respectively. ${ }^{10}$ In an Italian university hospital, 49.51\% of 103 hospitalized COVID-19 patients complained of sleep disturbance without any sex difference. It should be noticed that symptoms of sleep disturbance appeared immediately after the admission, and the frequency increased from $36.36 \%$ on the first 2 days to $69.23 \%$ after 7-day hospitalization, ${ }^{9}$ indicating that sleep disturbance in hospitalized COVID-19 patients cannot be simply explained by acute psychological response to the disease. Sleep disturbance was also found in mild patients even in mobile cabin hospitals, of whom more than two-thirds experienced insomnia on entry, but the overall insomnia levels (based on Insomnia Severity Index, ISI scores) were improved before discharge. ${ }^{6}$

Sleep disturbance may be associated with the adverse health consequences of COVID-19 patients. Compared to those without sleep disturbance, COVID-19 patients who suffered from sleep disturbance for at least 2 weeks during hospitalization presented with a slower recovery from lymphopenia, an increase in the deterioration of neutrophil-to-lymphocyte ratio. They also had a higher incidence of hospital-acquired infection, longer hospitalization, and an increased need for ICU care than those without sleep disturbance. ${ }^{12}$ The findings indicated the negative impacts of a sustained period of sleep disturbance on the delay in recovery of immune dysfunction in COVID-19 patients.

\section{Discharged Patients}

Sleep disturbance continued to bother $29.5-40 \%$ of COVID-19 survivors during the early post-discharge period, as reported in 4 studies $^{13-15}$ (Table 2). Up to $29.5 \%$ of 370 Chinese survivors complained of sleep disturbance during a median time of 22 days after discharge. ${ }^{13} \mathrm{~A}$ comparable proportion of 734 COVID-19 survivors (30.6\%) from Bangali reported insomnia, disturbance in sound sleep, and nightmares, ${ }^{16}$ while the prevalence (40\%) was higher in an Italian study. ${ }^{14}$ A French study showed that $30.8 \%$ of COVID-19 survivors still suffered from sleep disturbance even 110 days after being discharged, with no difference between ward- and ICU patients, ${ }^{15}$ highlighting the need for a long-term follow-up for sleep and rehabilitation consultants.

\section{Patients with Preexisting Sleep Disturbance}

Preexisting sleep disturbance might increase the susceptibility of COVID-19. ${ }^{17}$ A recent cross-sectional study showed that up to $60 \%$ of the patients reported sleep problems and had been taking sleeping pills over the past 12 months, ${ }^{18}$ indicating a high rate of preexisting sleep problems in COVID-19 patients and a possible role of poor sleep on the susceptibility of COVID-19. Cruz and colleagues have proposed a hypothesis that dysregulation of circadian rhythm and sleep may be associated with increased risk of SARS-CoV-2 infection and the severity of its clinical manifestations. ${ }^{17}$

The preexisting obstructive sleep apnea (OSA) and obesity hypoventilation syndrome are common co-morbid diseases in COVID-19 patients. The prevalence of OSA in COVID-19 patients was reported in 6 studies $^{19-24}$ (Table 3). In severe COVID-19 patients, the prevalence of OSA reached $21-28.6 \% .^{20,21}$ More recently, Perger and colleague conducted sleep tests in 44 COVID-19 patients, and identified $34 \%$ with OSA and $41 \%$ with central sleep apnea (CSA). Multivariate analysis revealed that higher BMI and higher obstructive AHI were associated with the need of ventilation support. ${ }^{22}$ COVID-19 patients with OSA are 1.58 times more likely to develop critical illness. ${ }^{23}$ The CORONADO study, which included 1317 hospitalized diabetic patients with COVID-19, also demonstrated that treated OSA prior to admission was associated with the 


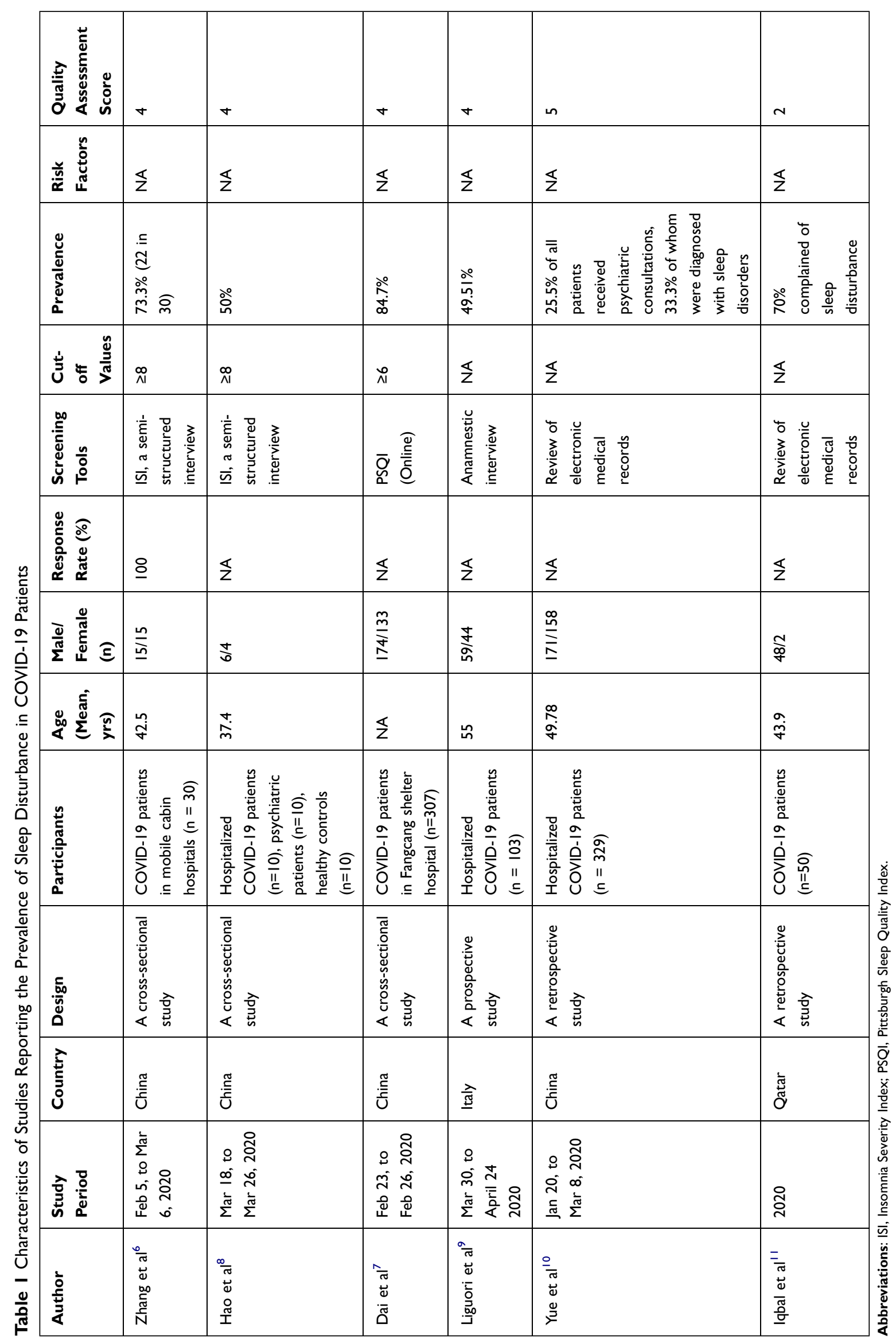




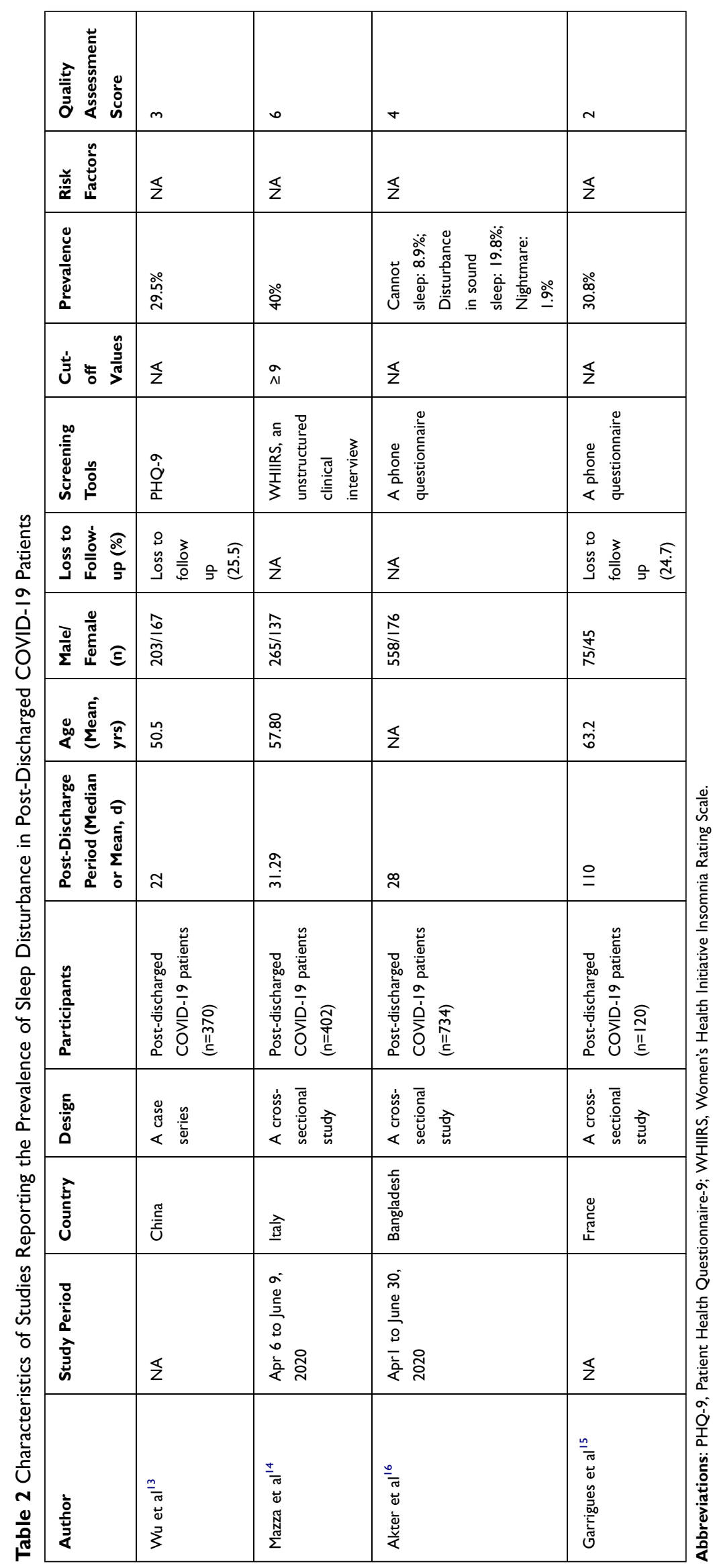




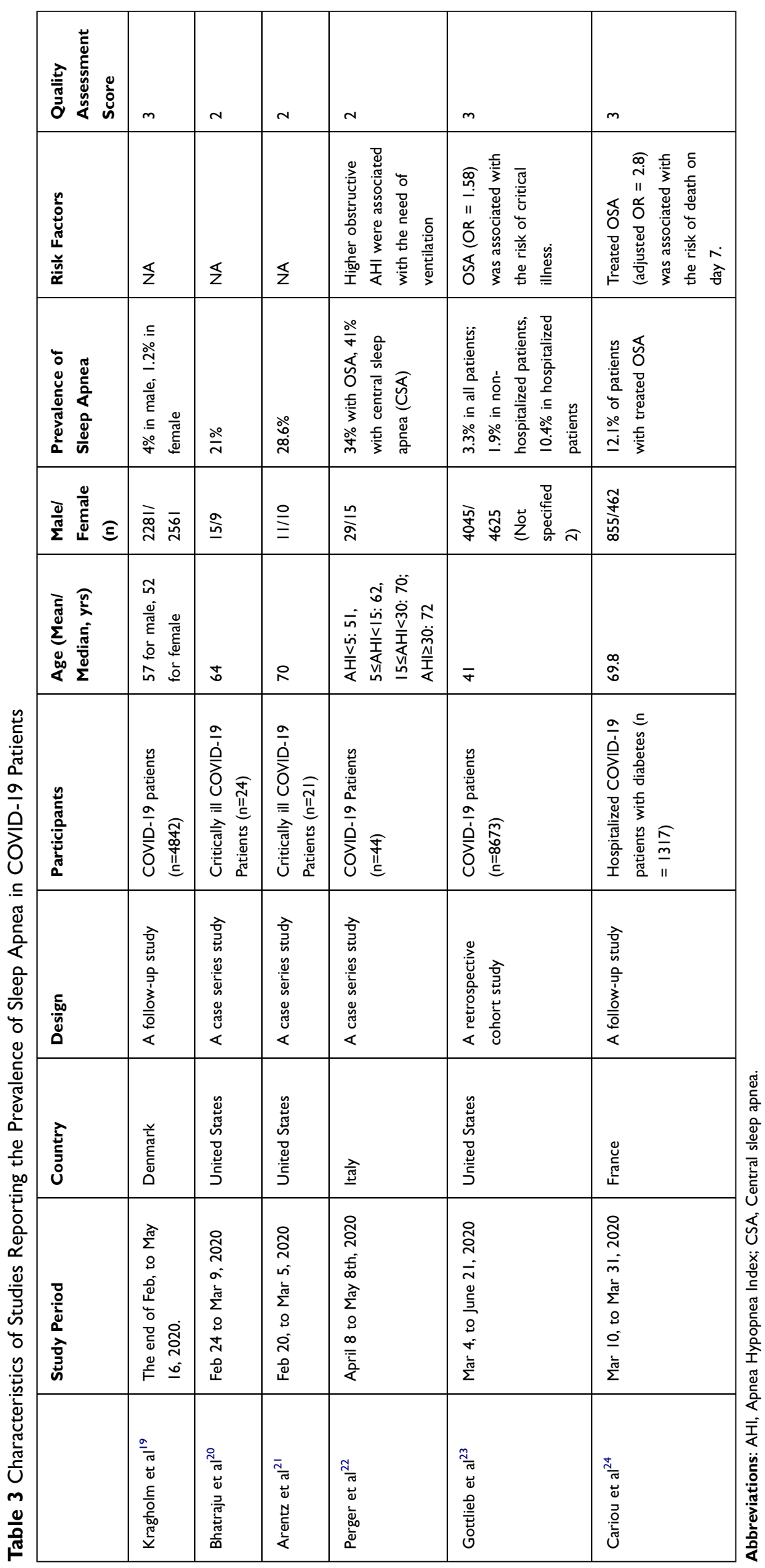


increased risk of death on day 7 (adjusted OR 2.65). ${ }^{24}$ Thus, it is possible that OSA is not simply a co-morbidity, but could be a risk factor for poor outcomes in COVID-19 patients. ${ }^{25,26}$ The plausible mechanistic pathways by which OSA may have adverse effects on OSA COVID-19 patients have been summarized in a previous review. ${ }^{2}$

\section{Factors Associated with Sleep Disturbance in COVID-19 Patients Physiologic Factors}

\section{Neuronal System Injury}

The neuronal injury directly and indirectly caused by SARSCoV-2 infection contributes to sleep disturbance in COVID-19 patients. SARS-CoV-2 could invade to the brain, possibly via the olfactory nerves or retrograde trans-synaptic dissemination from the lung to the medullary cardiorespiratory center. ${ }^{27,28}$ SARS-CoV-2 then rapidly spread to specific brain areas including thalamus and brain stem, which play essential roles in sleep control and respiratory regulation, respectively, and thereby increase the risks of abnormal sleep-wake behaviors and SDB. SARS-CoV-2 is also capable of causing secondary neuronal injury due to aberrant innate immune response, leading to chronic neurological sequelae that adversely affect sleep, emotion regulation, pain sensitivity, and energy. ${ }^{29,30}$ This indicates a possible long-lasting impact of COVID-19 on sleep. Additionally, the binding of SARS-CoV-2 to the ACE2expressing endothelial cells together with hypercoagulation status may contribute to the increased risk of cerebrovascular events, which contribute to sleep disturbance including inversed sleep-wake cycle, sleep-disordered breathing (SDB), and increased paradoxical sleep. ${ }^{31}$

\section{Symptoms, Severity of COVID-19, and Medication}

Except for the neuronal pathology caused by the virus, the physical discomforts including cough, fever, pain, and dyspnea may also destroy sleep. Relief of symptoms help to improve sleep in COVID-19 patients. Jiang and colleagues showed that Pittsburgh Sleep Quality Index (PSQI) scores were associated with subjective perception of the disease severity in COVID-19 patients. $^{32}$ Yang and colleagues recently found that scores of PSQI were positively associated with severity of pneumonia, and improvement of PSQI scores were positively related to improvement from COVID- $19 .^{33} \mathrm{To}$ be noted, adverse effects of medication, eg the use of corticosteroids, sedatives, beta-blocker, and nonsteroidal antiinflammatory drugs (NSAID) also create and exacerbate sleep problems in COVID-19 patients. Appropriate timing of medication, also called chronotherapy, should be taken into consideration to better fit patients' circadian rhythms and to minimize the side effects of medication on sleep eg. the use of corticosteroids, sedatives, beta-blocker, and nonsteroidal antiinflammatory drugs (NSAID) also create and exacerbate sleep problems in COVID-19 patients. Appropriate timing of medication, also called chronotherapy, should be taken into consideration to better fit patients' circadian rhythms and to minimize the side effects of medication on sleep. ${ }^{34}$

\section{Psychological Factors}

Sleep disturbance could also occur as the result of the psychologically traumatic effects of COVID-19. Two-week psychological intervention was able to improve PSQI scores, indicating a relationship between sleep disturbance and mental health in COVID-19 patients. ${ }^{33}$ Studies have demonstrated a high prevalence of mental health disorders in hospitalized and discharged patients with COVID-19 due to the fear of the new fatal virus infection, uncertainty about disease progression, worries about physical disability, loneliness and social isolation. ${ }^{8,10,13}$ Sleep is usually reciprocally associated with mental health. Sleep disturbance, and mental health disorders like depression, anxiety, and PTSD not only share symptoms, but also form a vicious cycle to deteriorate the prognosis in patients with COVID-19.Post-traumatic stress disorder (PTSD) not only share symptoms, but also form a vicious cycle to deteriorate the prognosis in patients with COVID-19.

\section{Environmental Factors}

Environmental factors including noise, abnormal light exposure, patient care activities, diagnostic and treatment procedures contribute to the ICU-related sleep disturbance. A previous study has indicated an innegligible role of environmental factors on sleep disturbance in hospitalized patients with COVID$19,{ }^{12}$ particularly for those critically ill patients. Sleep disturbance occurs frequently in ICU patients, presenting with decreased sleep efficiency, a shift toward light stages of sleep, increased arousals, and abnormal circadian rhythmicity. ${ }^{35}$

Taken together, physiologic and psychological traumatic effects of the infection may interact with environmental factors to increase the risk of sleep disturbance in COVID-19 patients. However, several questions remain to be solved. How does sleep change during the acute infection of COVID-19 and what is the patho-physiological mechanism? What is the relationship between sleep disturbance and occurrence and prognosis of COVID-19? Does sleep interference improve the prognosis of COVID-19? Moreover, yet little is known about long-term impacts of COVID-19 on sleep. A recent meta-analysis demonstrated a decrease in the frequency of 
insomnia from $41.9 \%$ (95\% CI, 22.5-50.5) during the acute illness to $12.1 \%(95 \% \mathrm{CI}, 8.6-16.3)$ after a follow-up duration varying from 60 days to 12 years in patients admitted to hospital for SARS or MERS. ${ }^{36}$ In the case of COVID-19, further studies are warranted to illustrate how long sleep disturbance would last after rehabilitation, and to what extent sleep disturbance could be improved over time.

\section{Sleep Disturbance in Healthcare Workers}

A total of 34 studies were included, with the subjective sleep quality being assessed by using self-reported questionnaires ${ }^{4,37-69}$ (Table 4). The prevalence of poor sleep quality in HWs during COVID-19 pandemic ranged from $18.4 \%$ to $84.7 \%$ based on scores of PSQI, ${ }^{4,37-47}$ which were comparable to that before the pandemic. ${ }^{70}$ A longitudinal study showed worsened sleep quality in 116 doctors and 99 nurses after one-month during the early COVID-19 outbreak, with a percentage of HWs with PSQI $>5$ increasing from $61.9 \%$ to $69.3 \%{ }^{44}$ The prevalence of sleep disturbance in HWs was generally higher than that in non-HWs or general population. ${ }^{53-55}$ Insomnia is the most prominent symptom with a prevalence ranging from $23.6 \%$ to $68.3 \%$ based on ISI scores. ${ }^{48-55,57,59-62}$ Moderate-to-severe insomnia with ISI $\geq 15$ presented in $6.78 \%-15 \%{ }^{48,50-52,54,55,57,59,61,62}$ Another two studies used AIS at a cut-off value of 6 showed $52.8 \%$ of nurses, ${ }^{65}$ and $68.3 \%$ of physicians ${ }^{66}$ suffered from insomnia.

Except for insomnia, symptoms of parasomnias including nightmares, sleepwalking, sleep terrors are more frequently reported in HWs than non-HWs. ${ }^{43}$ Notably, Zhuo and colleagues carried out a study to investigate overnight sleep in $26 \mathrm{HW}$ with insomnia using medical ring-shaped pulse oximeters, and showed that the incidence of comorbid moderate to severe sleep apnea in insomnia HW reached $38.5 \%$, indicating a high comorbidity rate of sleep apnea and insomnia attributable to stress.HWs with insomnia using medical ringshaped pulse oximeters, and showed that the incidence of comorbid moderate to severe sleep apnea in insomnia HWs reached $38.5 \%$, indicating a high comorbidity rate of sleep apnea and insomnia attributable to stress. ${ }^{71}$

\section{Factors Linked to Sleep Disturbance of HWs High Workloads}

High daily workloads contribute to poor sleep in HWs. Increased working hours were associated higher risk of sleep disturbance. ${ }^{53,58}$ The intensity of physical activity during daily work was negatively associated with sleep duration, and was positively associated with the feeling of tiredness during the wake-up in the morning. ${ }^{72}$
Being a shift worker has been reported to have 3.48 times likelihood to experience insomnia in the battle against COVID-19. ${ }^{43}$ Irregular and prolonged work shifts disrupt homeostatic and circadian rhythms and cause disturbance of several hormones, including melatonin and cortisol, leading to insufficient or inadequate sleep. Shift work not only impairs daytime function, and increases the risk of critical errors in HWs at work, ${ }^{73}$ but may also make HWs themselves more prone to COVID-19 infection. ${ }^{74,75}$ A recent singlecenter, retrospective study showed that implementation of new night shift schedule, changing from a four-day cycle to only daytime work for doctors with emergency techniques and extensive first aid experience and a six-day cycle in other doctors and nurses, significantly decreased the mortality of critically ill patients with COVID-19. ${ }^{76}$ Thus, more reasonable shift working schedules that allow for adequate rest for HWs and at the same time, ensure the continuity of treatments for patients, should be highly recommended during COVID-19 emergency status. ${ }^{76,77}$

\section{Occupation-Related Factors}

Several occupation-related factors contribute to the increased risk of sleep disturbance in HWs. The frontline HWs who are engaged in direct diagnosis, treatment, and care of COVID-19 patients, ${ }^{41,45,46,48-52,54,60,63,65,66}$ were more likely to experience sleep disturbance. Being a nurse is a risk factor for sleep disturbance (OR:1.48 to 3.132 ), ${ }^{41,46,62}$ while being a doctor was 0.44 times less likely to develop insomnia. ${ }^{49}$ The results were consistent with a previous study showing lower scores on posttraumatic stress in doctors than in nurse during SARS outbreak. ${ }^{78}$ However, being consultants and physicians, who took more responsibility on treating COVID-19 patients, were associated with sleep disturbance. ${ }^{4,47}$ The differences may be due to the fact that nurses are more likely to have more intense workloads, more frequent shift works, and more direct contacts with COVID-19 patients than doctors. ${ }^{49}$ Education and working experience are also closely associated with sleep problems in HWs. HWs with lower education level, ${ }^{49}$ less work experience, ${ }^{50,66}$ and poorer knowledge of crisis response, ${ }^{41}$ and who were lack of sufficient protective equipment ${ }^{57}$ had higher probability of experiencing sleep problems.

\section{Psychological Factors}

Psychological factors were associated with sleep disturbance in HWs, including psychological symptoms (ie depression and anxiety), ${ }^{29,37-39,44,45,58,66}$ COVID-19related bereavement, ${ }^{45,64}$ worries about the COVID-19 outbreak, ${ }^{49,65}$ being worried about being infected, ${ }^{49}$ 


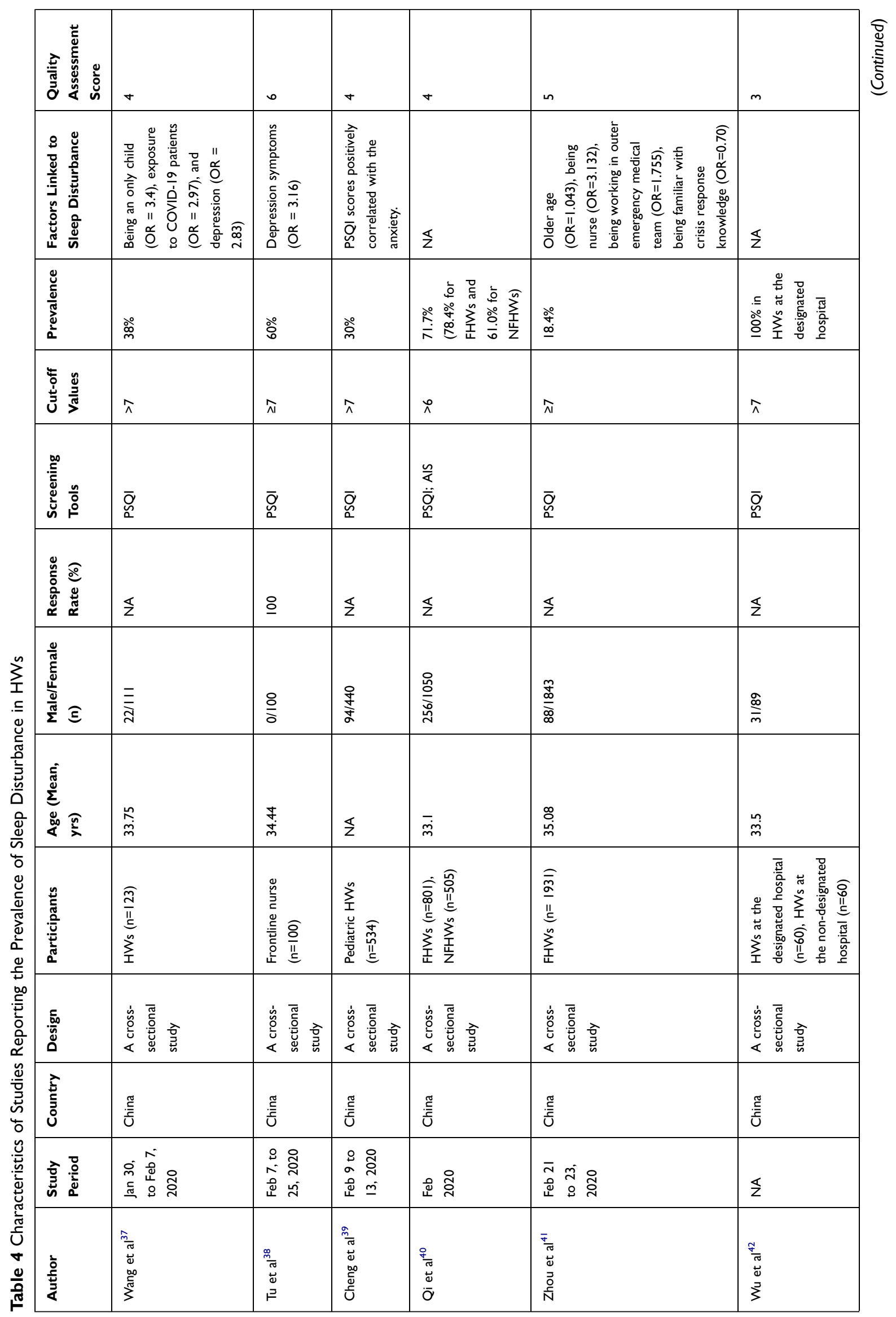




\begin{tabular}{|c|c|c|c|c|}
\hline 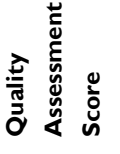 & $\sigma$ & + & 0 & in \\
\hline 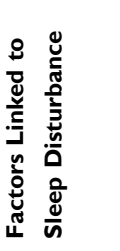 & 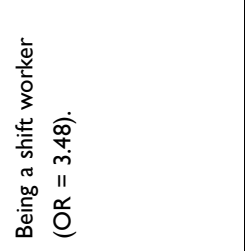 & 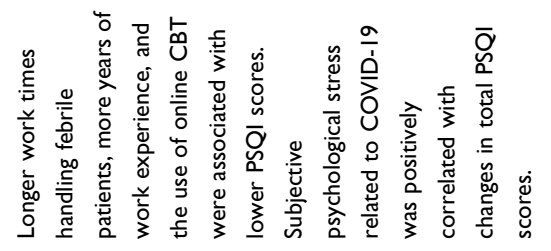 & 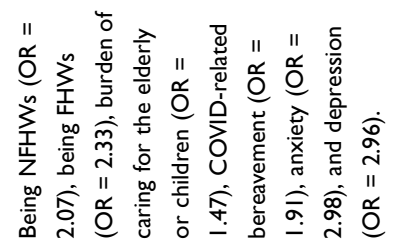 & 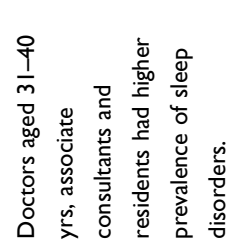 \\
\hline & 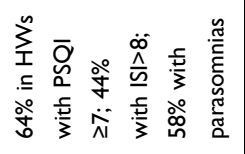 & 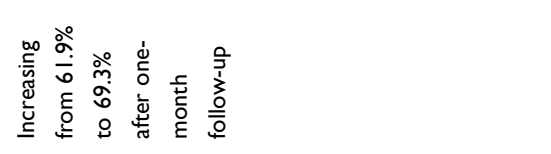 & $\frac{\circ}{\stackrel{\circ}{6}}$ & $\begin{array}{l}\stackrel{\circ}{\sigma} \\
\stackrel{\gamma}{\gamma}\end{array}$ \\
\hline 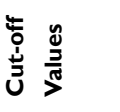 & 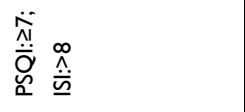 & $\stackrel{n}{\Lambda}$ & $\stackrel{\text { L }}{\wedge}$ & $\stackrel{n}{\wedge}$ \\
\hline 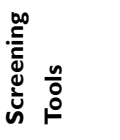 & $\begin{array}{l}\overline{\bar{s}} \\
\overline{\bar{o}} \\
\underline{0}\end{array}$ & $\overline{\mathrm{g}}$ & 啇 & $\overline{\mathrm{O}}$ \\
\hline 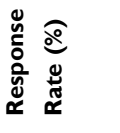 & ○े & 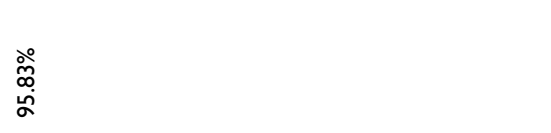 & $\begin{array}{l}\stackrel{\circ}{\circ} \\
\stackrel{\infty}{\circ}\end{array}$ & $\mathbb{z}$ \\
\hline 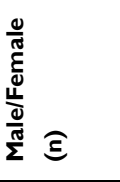 & $\frac{8}{2}$ & 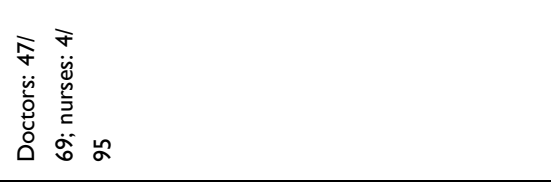 & 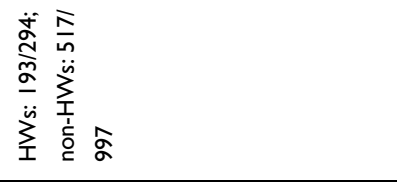 & $\underset{\substack{\text { N } \\
\stackrel{\sim}{N}}}{ }$ \\
\hline 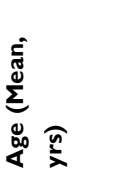 & 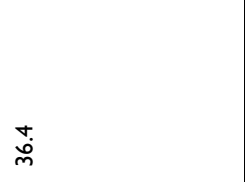 & 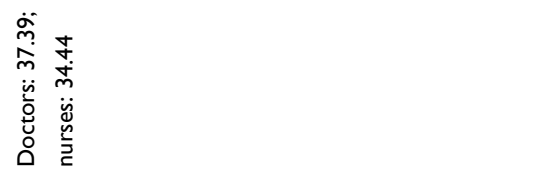 & 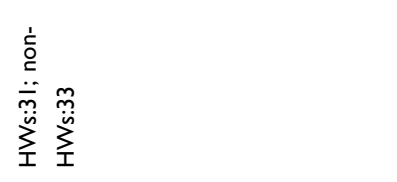 & $\Sigma$ \\
\hline 产 & 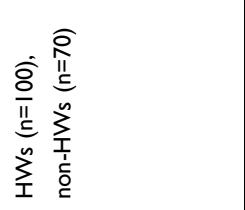 & 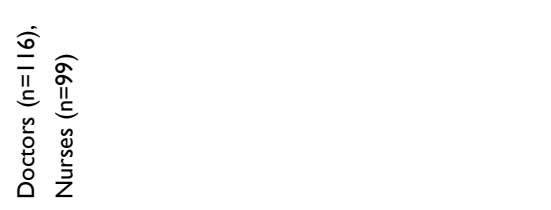 & 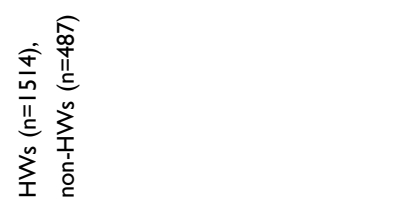 & 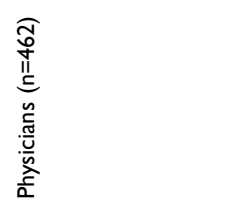 \\
\hline פ. & 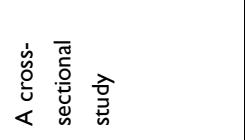 & 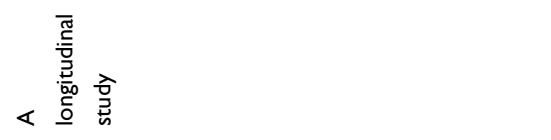 & 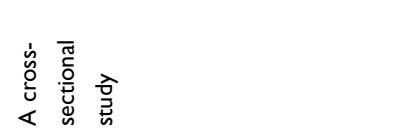 & 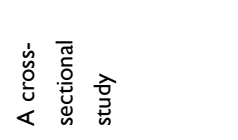 \\
\hline نे & 䓌 & 莺 & 牟 & 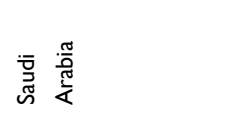 \\
\hline 希 & 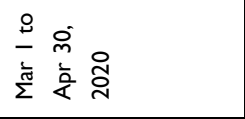 & 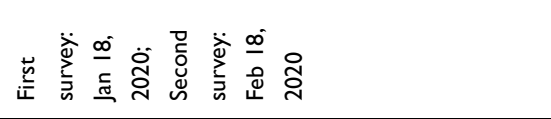 & 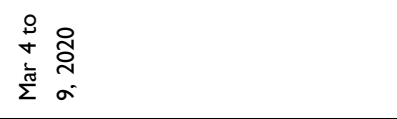 & 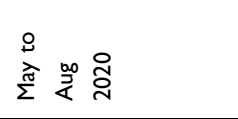 \\
\hline 高 & 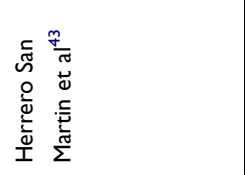 & 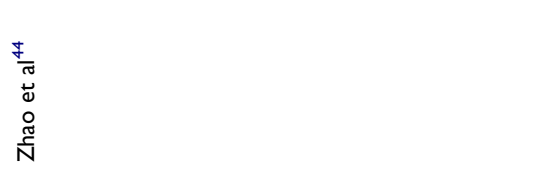 & 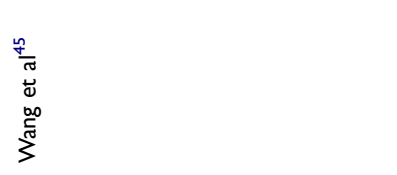 & 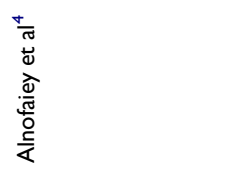 \\
\hline
\end{tabular}




\begin{tabular}{|c|c|}
\hline in & $\sigma$ \\
\hline 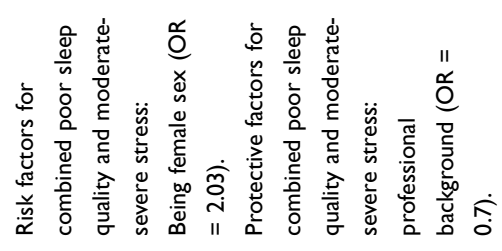 & 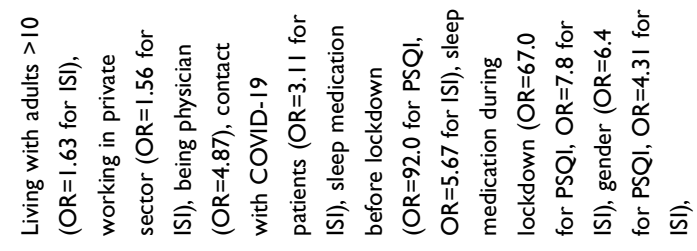 \\
\hline خั่ & 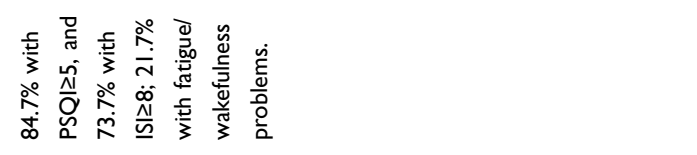 \\
\hline$\stackrel{\sim}{\Lambda}$ & 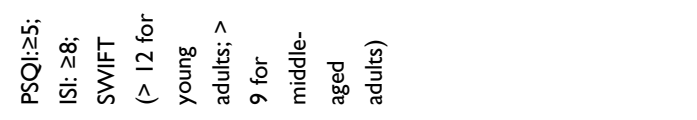 \\
\hline$\overline{\mathrm{o}}$ & 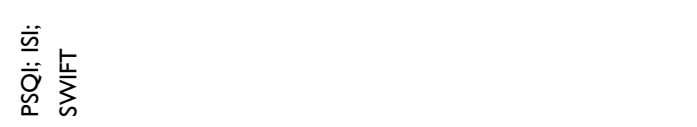 \\
\hline ஓें & $\mathbb{z}$ \\
\hline$\stackrel{\infty}{\stackrel{\infty}{k}}$ & 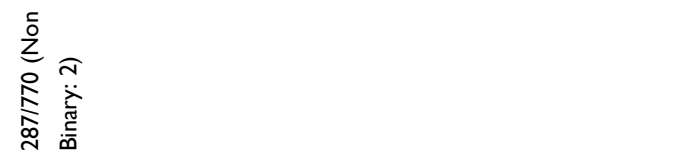 \\
\hline ָ̃ & $\hat{\bar{\sigma}}$ \\
\hline 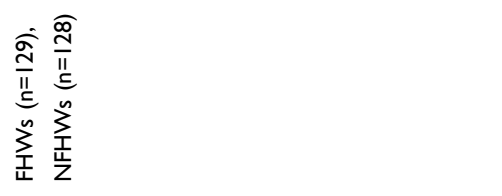 & 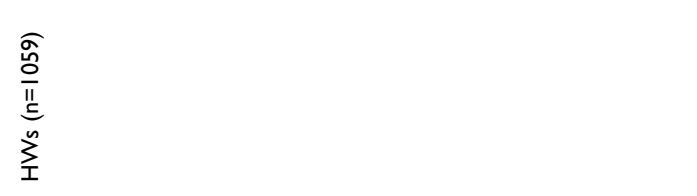 \\
\hline 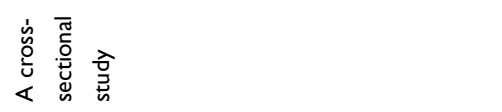 & 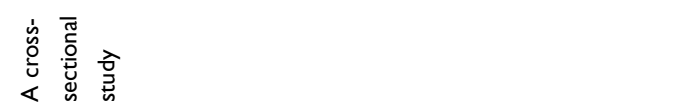 \\
\hline 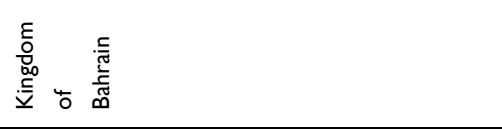 & 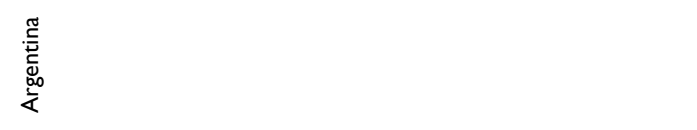 \\
\hline 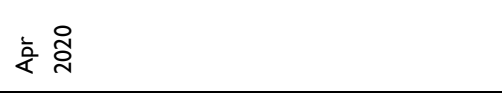 & 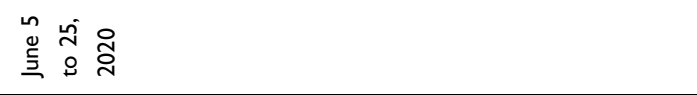 \\
\hline 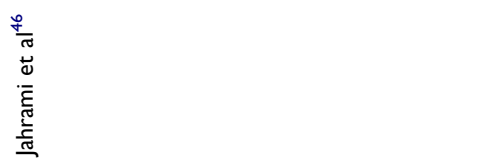 & 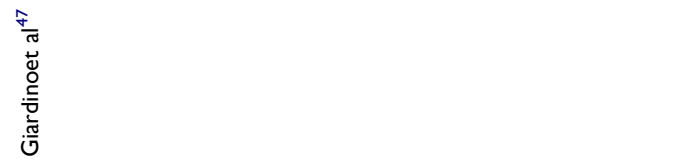 \\
\hline
\end{tabular}




\begin{tabular}{|c|c|c|}
\hline 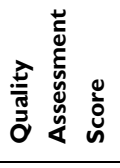 & 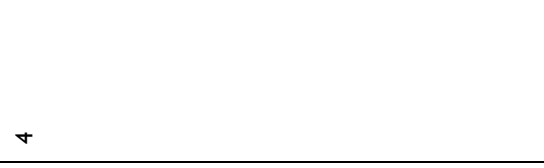 & in \\
\hline 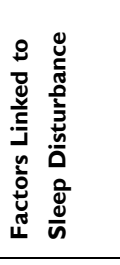 & 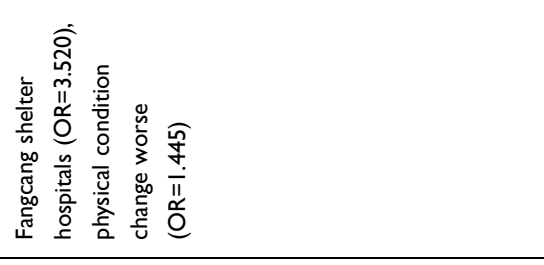 & 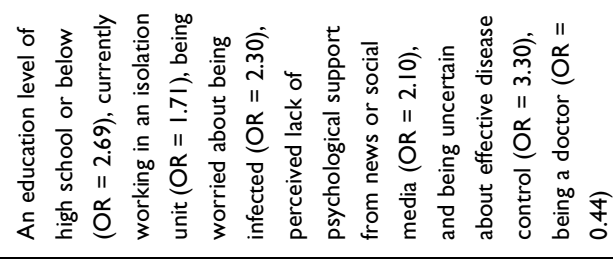 \\
\hline 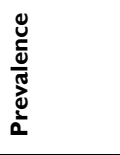 & 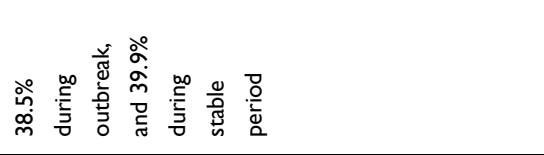 & $\frac{\circ}{\grave{\rho}}$ \\
\hline 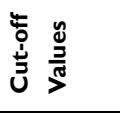 & $\stackrel{\infty}{\wedge}$ & $\stackrel{\infty}{\wedge}$ \\
\hline 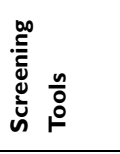 & $\underline{\bar{n}}$ & $\underline{\bar{n}}$ \\
\hline 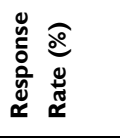 & $\mathbb{z}$ & $\mathbb{z}$ \\
\hline 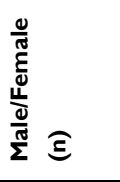 & 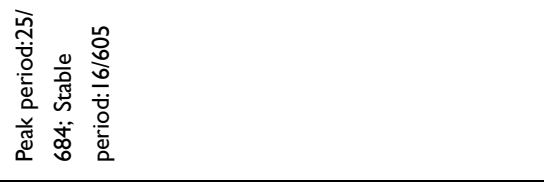 & $\underset{\substack{\hat{\sigma} \\
\text { a }}}{\bar{\sigma}}$ \\
\hline 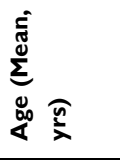 & 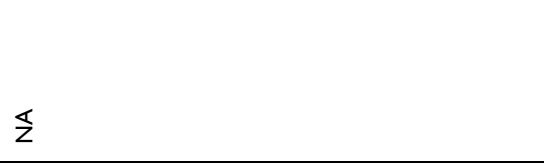 & $\S$ \\
\hline 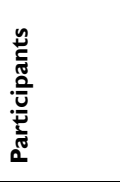 & 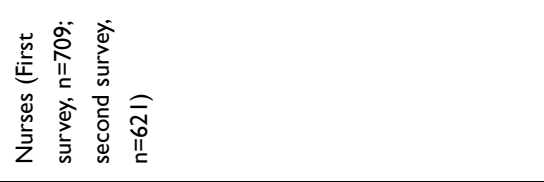 & 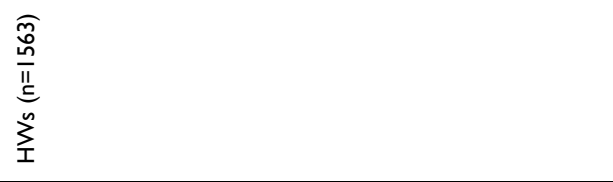 \\
\hline$\frac{.5}{\overline{0}}$ & 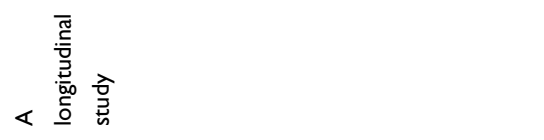 & 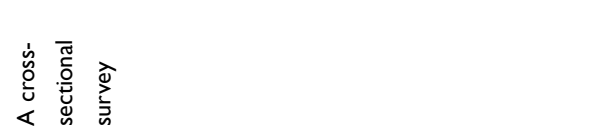 \\
\hline 害 & 㞼 & 祍 \\
\hline 空 & 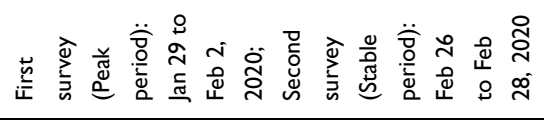 & 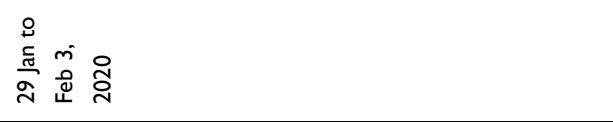 \\
\hline $\begin{array}{l}\frac{1}{2} \\
\frac{1}{3} \\
\frac{3}{4}\end{array}$ & 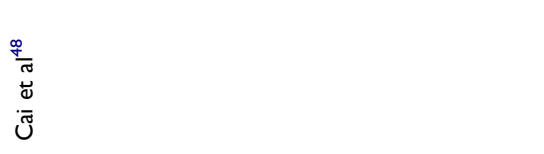 & 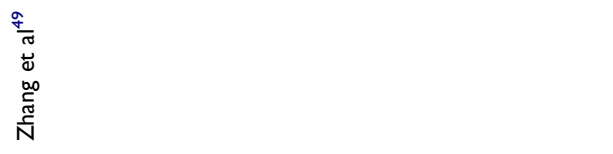 \\
\hline
\end{tabular}




\begin{tabular}{|c|c|c|c|}
\hline 0 & 。 & $\circ$ & 。 \\
\hline 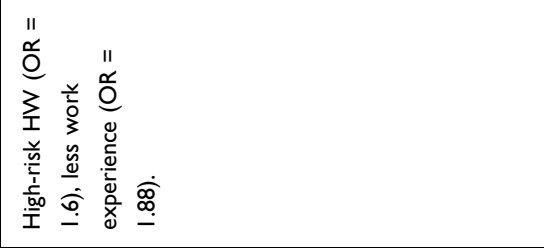 & 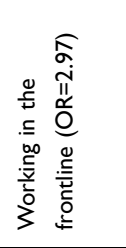 & 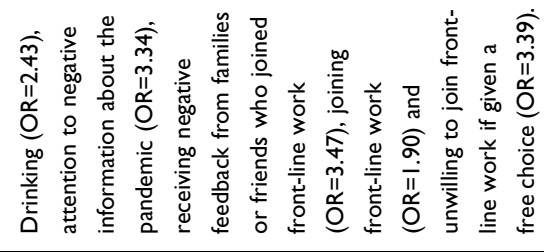 & 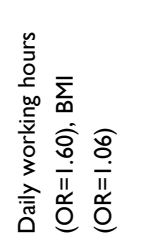 \\
\hline \begin{tabular}{|l}
$\stackrel{\circ}{\circ}$ \\
$\dot{\sigma}$
\end{tabular} & $\begin{array}{l}\text { ○े } \\
\text { ఫे }\end{array}$ & 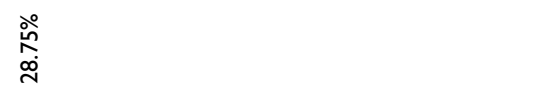 & 今ें \\
\hline 文 & $\stackrel{\infty}{\wedge}$ & $\bigwedge_{\Lambda}^{\infty}$ & $\stackrel{\infty}{\wedge}$ \\
\hline$\underline{\bar{n}}$ & $\underline{\bar{s}}$ & $\underline{\bar{n}}$ & $\underline{\bar{s}}$ \\
\hline 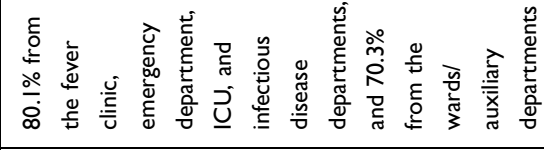 & $\begin{array}{l}\stackrel{\circ}{\circ} \\
\text { ô } \\
0\end{array}$ & $\mathbb{z}$ & $\mathbb{z}$ \\
\hline 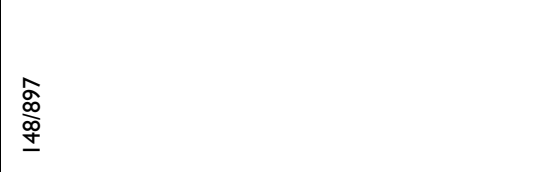 & 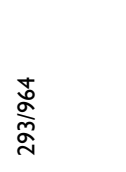 & 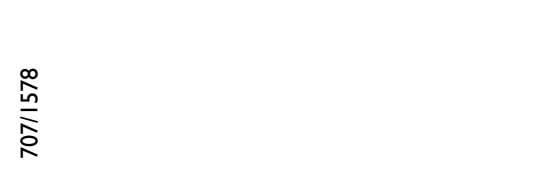 & 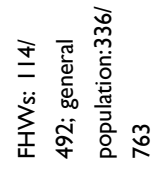 \\
\hline $\mathbb{z}$ & $\mathbb{z}$ & $\frac{\stackrel{\circ}{m}}{m}$ & 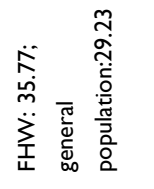 \\
\hline 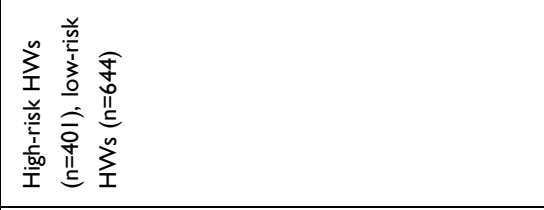 & 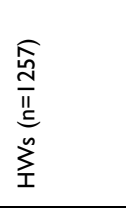 & 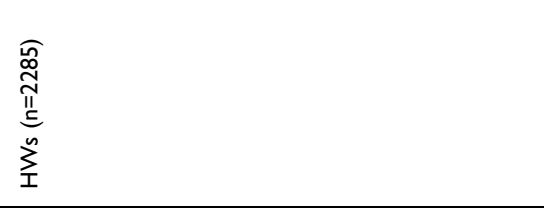 & 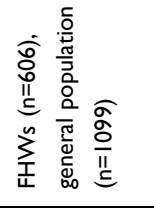 \\
\hline 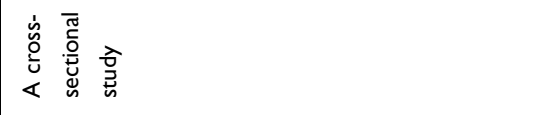 & 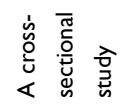 & 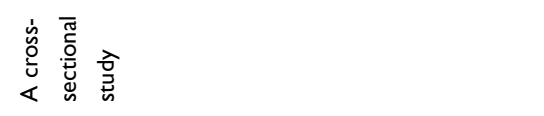 & 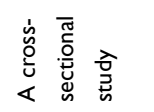 \\
\hline 胥 & 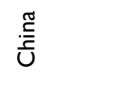 & 祍 & 疍 \\
\hline 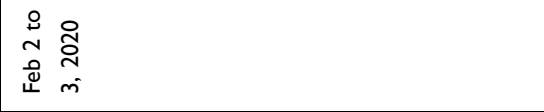 & 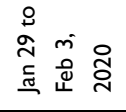 & 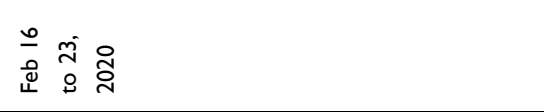 & 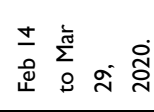 \\
\hline 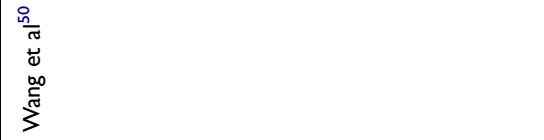 & 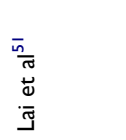 & 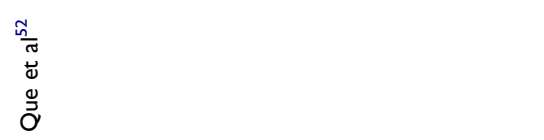 & $\begin{array}{l}\frac{m_{0}}{\pi} \\
\frac{0}{0} \\
\frac{\partial}{N} \\
\frac{N}{N}\end{array}$ \\
\hline
\end{tabular}




\begin{tabular}{|c|c|c|c|c|}
\hline 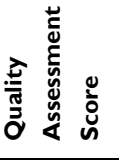 & 0 & 0 & 0 & 0 \\
\hline 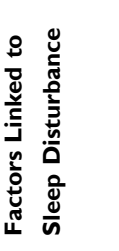 & 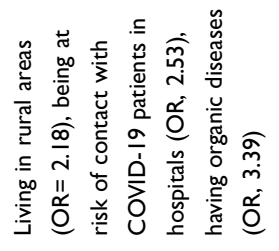 & $\S$ & 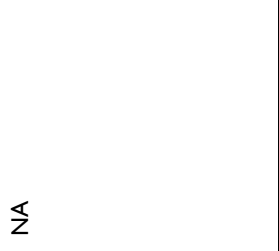 & 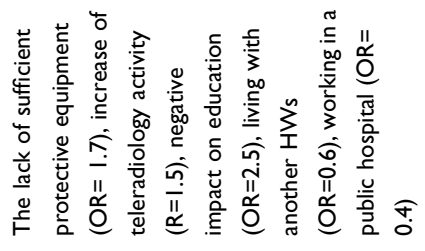 \\
\hline 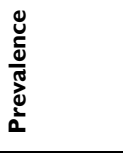 & $\begin{array}{c}\stackrel{\circ}{\circ} \\
\substack{\infty \\
\text { j. }} \\
\end{array}$ & 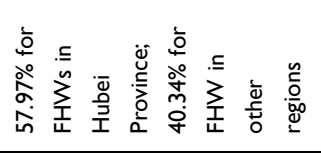 & 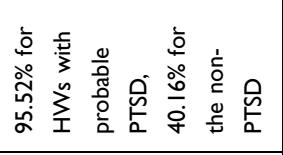 & $\begin{array}{l}\stackrel{\circ}{\circ} \\
\text { 广े } \\
\end{array}$ \\
\hline 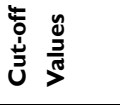 & $\stackrel{\infty}{\wedge}$ & $\stackrel{\infty}{\Lambda}$ & $\stackrel{\infty}{\Lambda}$ & $\stackrel{\infty}{\wedge}$ \\
\hline 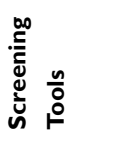 & $\underline{\bar{s}}$ & $\underline{\bar{n}}$ & $\underline{\bar{s}}$ & $\underline{\bar{s}}$ \\
\hline 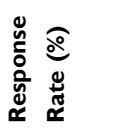 & 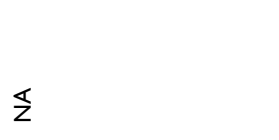 & 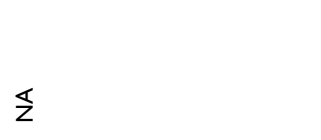 & $\S$ & $\stackrel{\circ}{\stackrel{\circ}{~}}$ \\
\hline 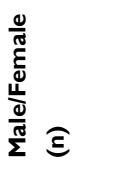 & 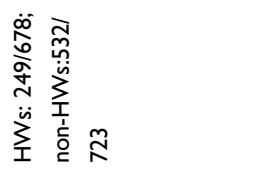 & 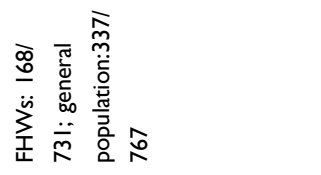 & 总 & 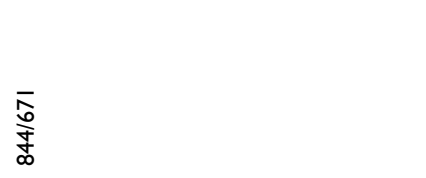 \\
\hline 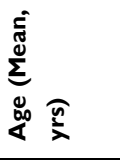 & 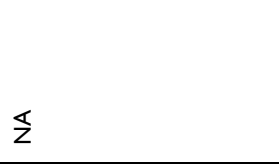 & 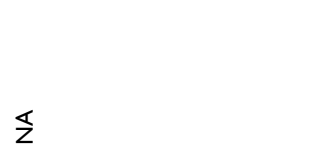 & 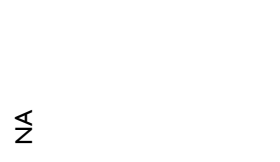 & $\S$ \\
\hline 芴 & 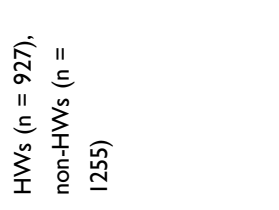 & 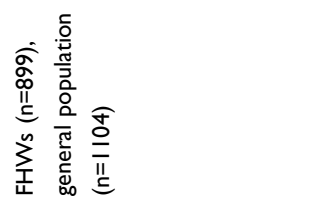 & 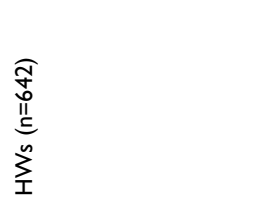 & 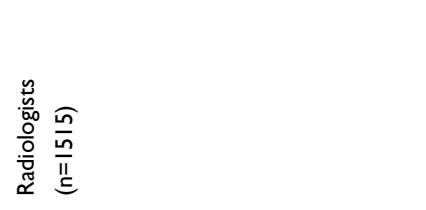 \\
\hline 高 & 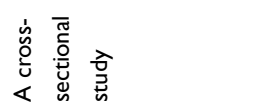 & 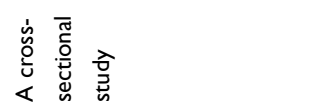 & 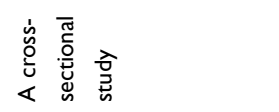 & 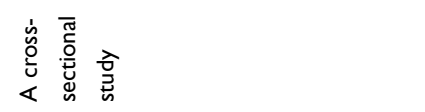 \\
\hline نे & 祍 & 茎 & 蛇 & 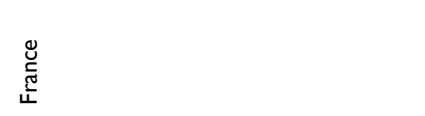 \\
\hline 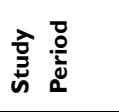 & 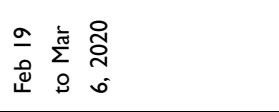 & 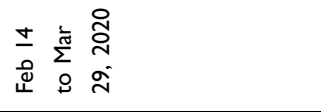 & 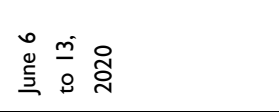 & 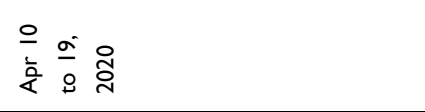 \\
\hline 高 & 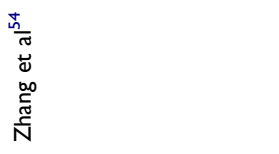 & 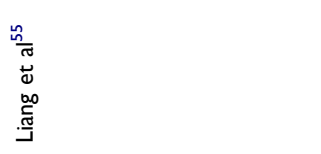 & 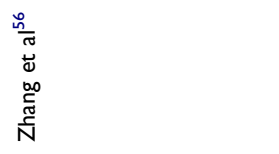 & 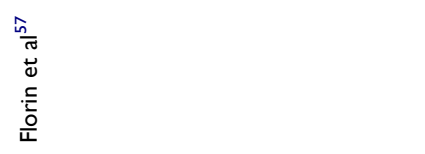 \\
\hline
\end{tabular}




\begin{tabular}{|c|c|c|c|c|}
\hline 0 & in & 0 & in & $\circ$ \\
\hline 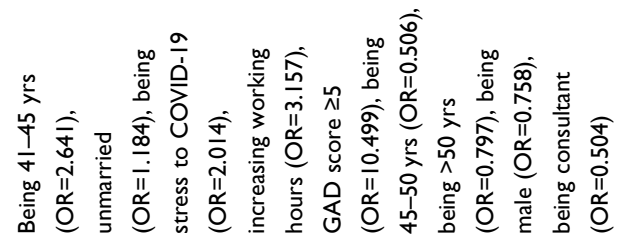 & $\S$ & 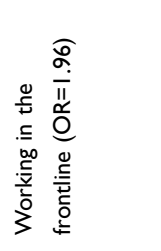 & 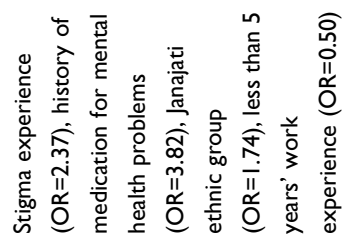 & 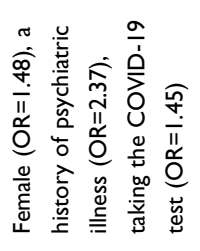 \\
\hline 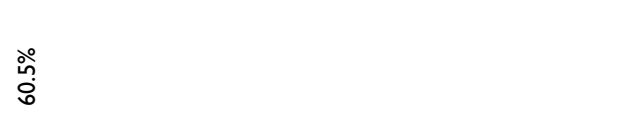 & $\frac{\stackrel{\circ}{\circ}}{\stackrel{+}{+}}$ & 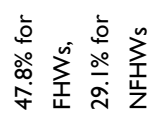 & $\begin{array}{l}\stackrel{\circ}{\circ} \\
\stackrel{m}{m}\end{array}$ & 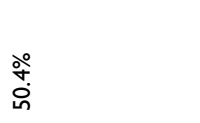 \\
\hline$\stackrel{\infty}{\wedge 1}$ & $\bigwedge_{\Lambda}^{\infty}$ & $\stackrel{a}{i}$ & $\frac{\circ}{\wedge}$ & $\frac{\circ}{\wedge}$ \\
\hline$\underline{\bar{n}}$ & $\underline{\bar{n}}$ & $\underline{\bar{n}}$ & $\underline{\bar{n}}$ & $\underline{\bar{x}}$ \\
\hline$\S$ & 官 & $\S$ & 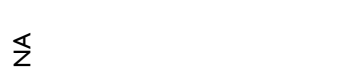 & $\mathbb{z}$ \\
\hline 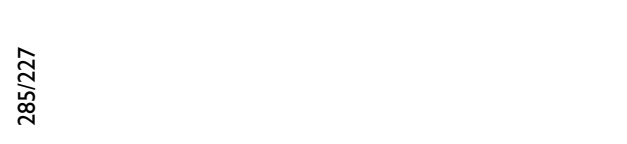 & 咅 & 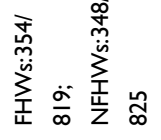 & 号 & $\frac{\text { Iิ }}{\frac{0}{m}}$ \\
\hline$\overleftarrow{z}$ & 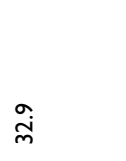 & 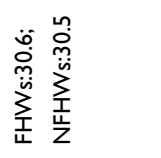 & $\stackrel{\sim ్ య}{\sim}$ & $\S$ \\
\hline 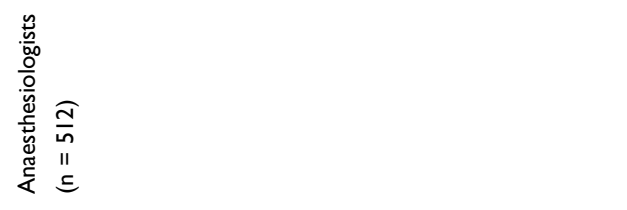 & 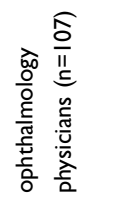 & 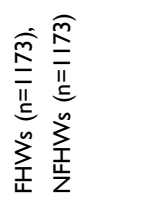 & 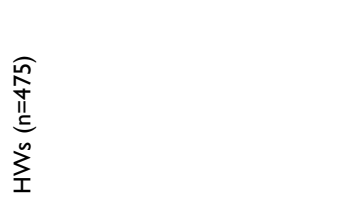 & 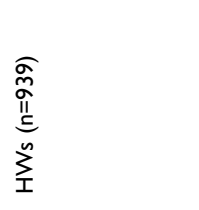 \\
\hline 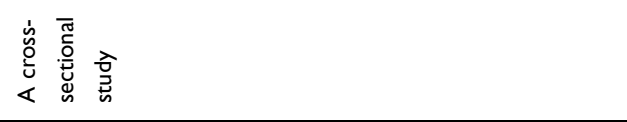 & 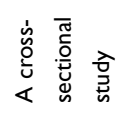 & 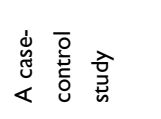 & 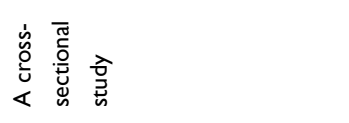 & 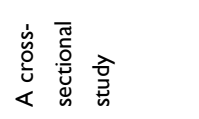 \\
\hline $\begin{array}{l}\text { 孚 } \\
\underline{\underline{\underline{n}}}\end{array}$ & 馬 & 䗆 & $\begin{array}{l}\overline{\bar{g}} \\
\text { zu }\end{array}$ & 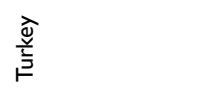 \\
\hline 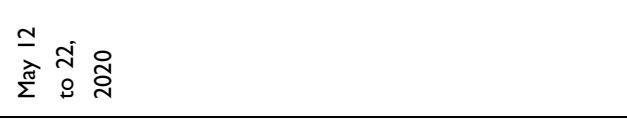 & 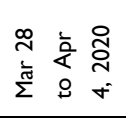 & 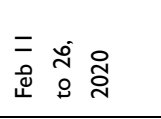 & 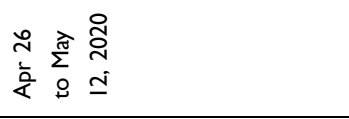 & 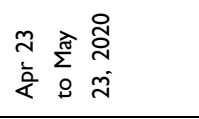 \\
\hline 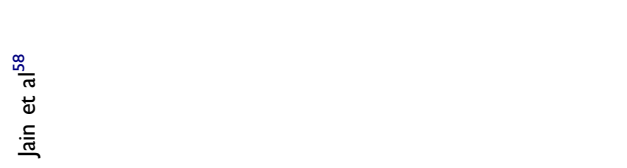 & 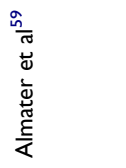 & 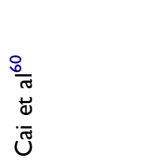 & 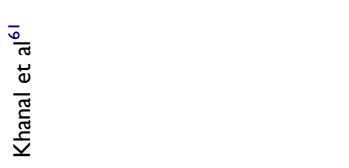 & 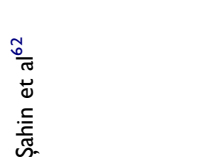 \\
\hline
\end{tabular}




\begin{tabular}{|c|c|c|c|}
\hline 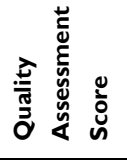 & $\circ$ & $\circ$ & $\circ$ \\
\hline 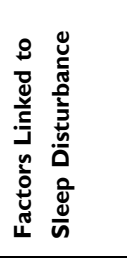 & 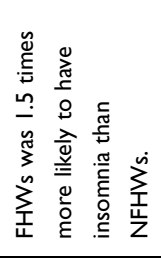 & 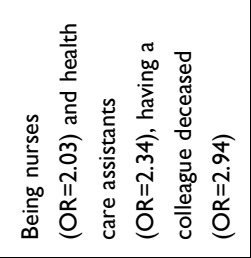 & 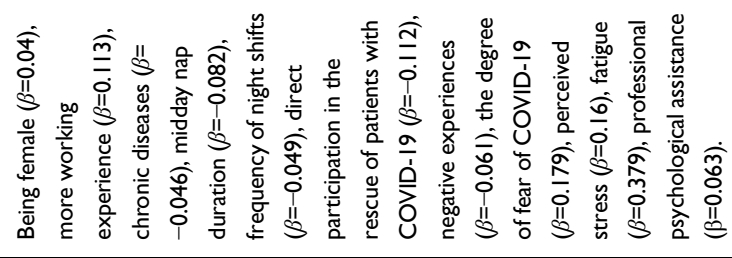 \\
\hline 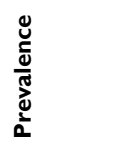 & $\begin{array}{l}\stackrel{\circ}{\circ} \\
\stackrel{0}{0}\end{array}$ & $\begin{array}{l}\stackrel{\text { సે }}{\infty} \\
\infty\end{array}$ & $\begin{array}{l}\stackrel{\circ}{\circ} \\
\text { ஸे }\end{array}$ \\
\hline 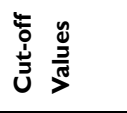 & $\frac{\nabla}{N}$ & $\widetilde{\Lambda}$ & $\stackrel{\circ}{\wedge}$ \\
\hline 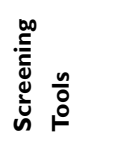 & $\underline{\bar{\sigma}}$ & $\underline{\bar{s}}$ & $\frac{\Omega}{\alpha}$ \\
\hline 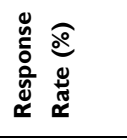 & 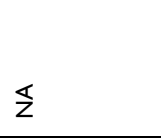 & $\mathbb{z}$ & $\mathbb{z}$ \\
\hline 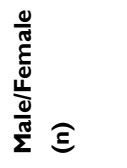 & 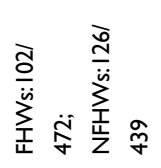 & $\frac{\mathbb{t}}{\frac{\mathrm{o}}{\mathrm{H}}}$ & $\frac{\text { P }}{\stackrel{\frac{9}{f}}{n}}$ \\
\hline 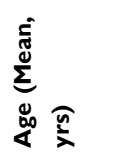 & 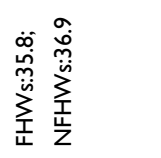 & 品 & $\mathbb{z}$ \\
\hline 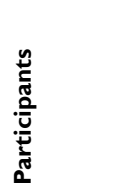 & 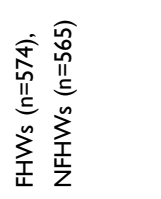 & 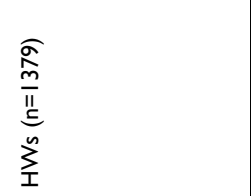 & 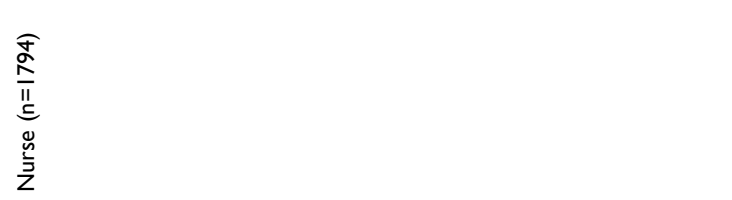 \\
\hline 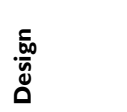 & 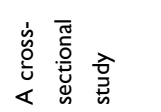 & 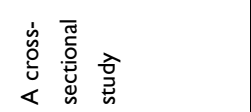 & 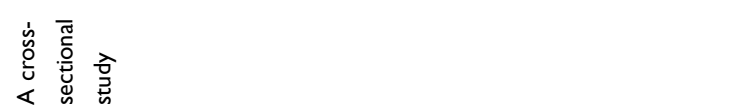 \\
\hline 害 & 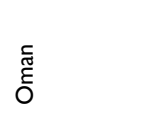 & 첲 & 莺 \\
\hline 窟 & 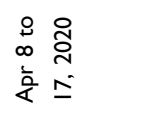 & 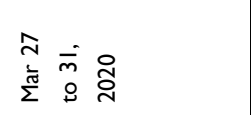 & 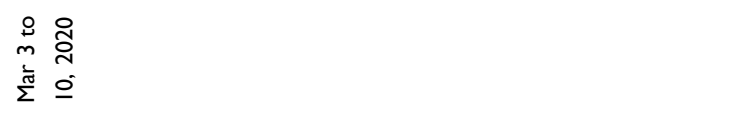 \\
\hline 高 & 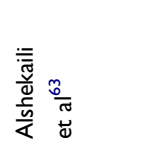 & 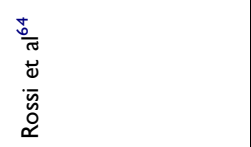 & 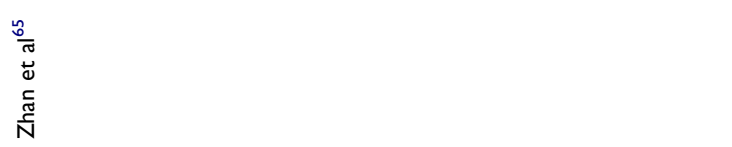 \\
\hline
\end{tabular}




\begin{tabular}{|c|c|c|c|}
\hline in & $m$ & + & $\sigma$ \\
\hline 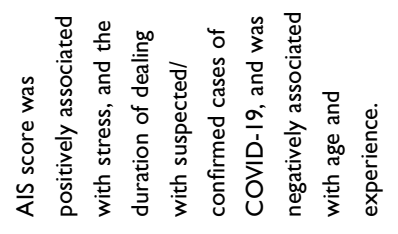 & 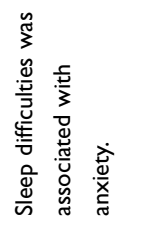 & 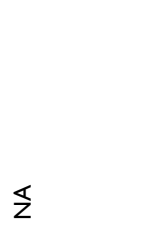 & $\S$ \\
\hline 产 & $\frac{\circ}{\stackrel{\text { }}{i}}$ & 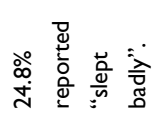 & 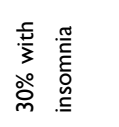 \\
\hline ו̊ & - & - & - \\
\hline$\frac{\tilde{\omega}}{\kappa}$ & 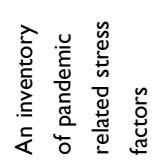 & 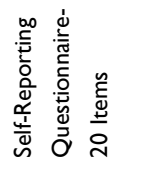 & 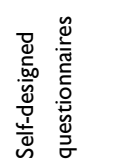 \\
\hline $\mathbb{z}$ & $\mathbb{z}$ & & $\mathbb{z}$ \\
\hline $\begin{array}{l}\stackrel{\bigcirc}{\infty} \\
\stackrel{\infty}{\infty} \\
\underline{\infty}\end{array}$ & $\begin{array}{l}\text { F } \\
\text { 点 } \\
\text { م⿱ }\end{array}$ & $\mathbb{z}$ & $\S$ \\
\hline $\begin{array}{l}\stackrel{\circ}{\circ} \\
\text { 㟧 }\end{array}$ & $\begin{array}{l}\hat{o} \\
\dot{f}\end{array}$ & $\mathbb{z}$ & $\mathbb{Z}$ \\
\hline 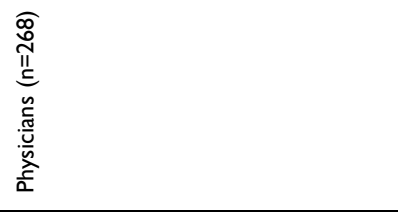 & 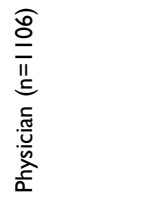 & 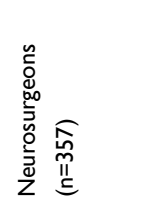 & 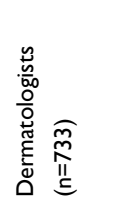 \\
\hline 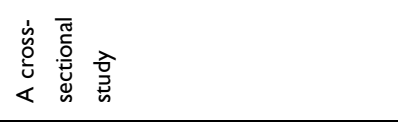 & 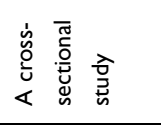 & 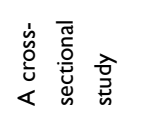 & 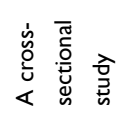 \\
\hline $\begin{array}{l}\overline{\bar{g}} \\
\end{array}$ & $\begin{array}{l}\overline{\bar{d}} \\
\underline{\underline{\underline{w}}} \\
\end{array}$ & 乞 & 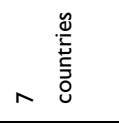 \\
\hline 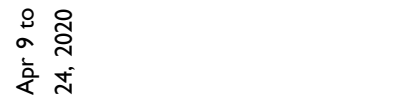 & 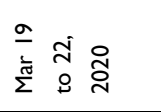 & $\S$ & 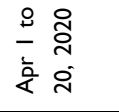 \\
\hline 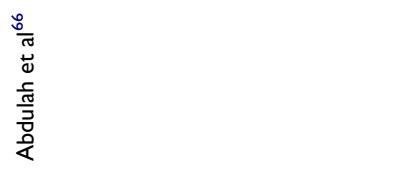 & 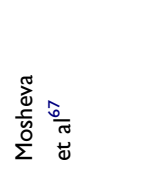 & 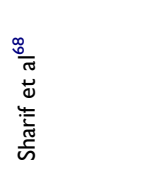 & 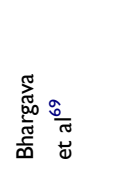 \\
\hline
\end{tabular}


perceived lack of psychological support, ${ }^{49}$ and preexisting psychological diseases or sleep medication. ${ }^{47,61,62}$ The relationship between sleep disturbance and COVID-19related stress may be bidirectional. On one hand, the stress associated with high risk of the virus infection and high patient mortality, perceived physical isolation, the necessity for constant vigilance regarding infection control procedures, and concern about family members could cause anxiety, and depression, ${ }^{46}$ and impair sleep quality. On the other hand, poor sleep may result in daytime fatigue, loss of interest, impairment of the daytime function, and increase the risk of critical errors at work, ${ }^{73}$ which in turn, worsens psychological condition in HWs.

Moreover, other factors including being female, ${ }^{62,65}$ being aged $41-45$ yrs, ${ }^{58}$ being the only child, ${ }^{37}$ having burden of caring for the elderly or children, ${ }^{45}$ physical condition and medication history, ${ }^{45,47,48,61,62}$ being unmarried, ${ }^{58}$ also contribute to sleep disturbance in HWs.

Taken together, high workloads may interact with COVID-19-related stress to increase the risk of sleep disturbance in HWs. The majority of the included studies were cross-sectional surveys showing the prevalence of sleep disturbance in HWs during the pandemic. Only one longitudinal study reported a slight increase in the prevalence of sleep disturbance. ${ }^{44}$ Further studies should be conducted to determine the prevalence of new-onset or worsened sleep disturbance during the pandemic. Additionally, the impacts of sleep disturbance on health being, life quality and working performance in HWs during and after the pandemic also need further investigation.

\section{Sleep Disturbance in the General Public}

The prevalence of sleep disturbance in the general population during COVID-19 pandemic was reported in 36 studies, ${ }^{79-114}$ ranging from $17.65 \%$ to $81 \%,{ }^{79-86} 24.66 \%$ to $86 \%,{ }^{87-89-93-95-96}$ and $30 \%$ to $56 \%,{ }^{100-103}$ based on scores of PSQI, ISI and AIS, respectively (Table 5), which were generally higher than that before the pandemic. ${ }^{83,115}$ However, the effects of COVID-19-related lockdown on the public sleep quality remain controversial. Data from Italian and Australian studies reported that approximately half of the participants experienced worsened sleep quality during the lockdown. ${ }^{84,85,107,108}$ Similarly, a study in China showed more than one-third of the participants had increased impaired sleep quality. ${ }^{91}$ Symptoms of sleep disturbance commonly overlapped with those of depression, anxiety and PTSD in the general public. $^{83}$ On the contrary, a longitudinal study in the
United State showed that $47 \%$ had improved sleep with longer sleep duration, and only $29 \%$ had worsened sleep from baseline to lockdown. ${ }^{116}$ Another multicenter study from 11 countries also showed a reduced prevalence of insomnia after 2-month lockdown. ${ }^{110}$ The results may indicate societal resilience to the chronic threat of viral infection and the changes of daily life. The varied proportions of sleep disturbance and its changes among the countries may be, at least partially, explained by the difference in epidemic control policy and the public attitude towards COVID-19 crisis. Interestingly, Kocevska and colleagues found that $20 \%$ of pre-pandemic good sleepers experienced worsened sleep, while a quarter of participants with pre-pandemic clinical insomnia experienced an amelioration of insomnia during COVID-19 pandemic.117 They argued that the effects of lockdown on sleep quality is not uniform, and emphasized the individual difference in response to COVID-19 crisis.

\section{Factors Linked to Sleep Disturbance of General Public}

Physiologic Factors

The circadian rhythm may be altered due to reduced exposure to sunlight, reduced physical activity and changes in working schedule during COVID-19 lockdown. However, the impacts of circadian rhythm alteration on sleep and other health consequences are controversial. On one hand, reduced social jetlag (driven by delayed mid-sleep on weekdays), reduced social sleep restriction (driven by increased sleep duration on weekdays) and decreased sleep debt may harmonize sleep schedules throughout the week, and thereby may limit the decline in sleep quality during the lockdown. ${ }^{118,119}$ On the other hand, later chronotype, manifested as delayed mid-sleep on weekdays, may be associated with increased risk of mood symptoms including depression, ${ }^{120}$ which in turn, may worsen sleep quality.

\section{Social-Psychological Factors}

The impacts of age on sleep during COVID-19 pandemic seem controversial. Two studies revealed that people aged more than 30 yrs are more likely to develop sleep disturbance during COVID-19 pandemic, ${ }^{88,106}$ consistent with previous studies showing that the prevalence increased with age. ${ }^{121,122}$ The age-related deterioration in sleep may be attributable to not only the effects of aging on circadian pacemaker and sleep structure but also the increased working and social stress that older people 


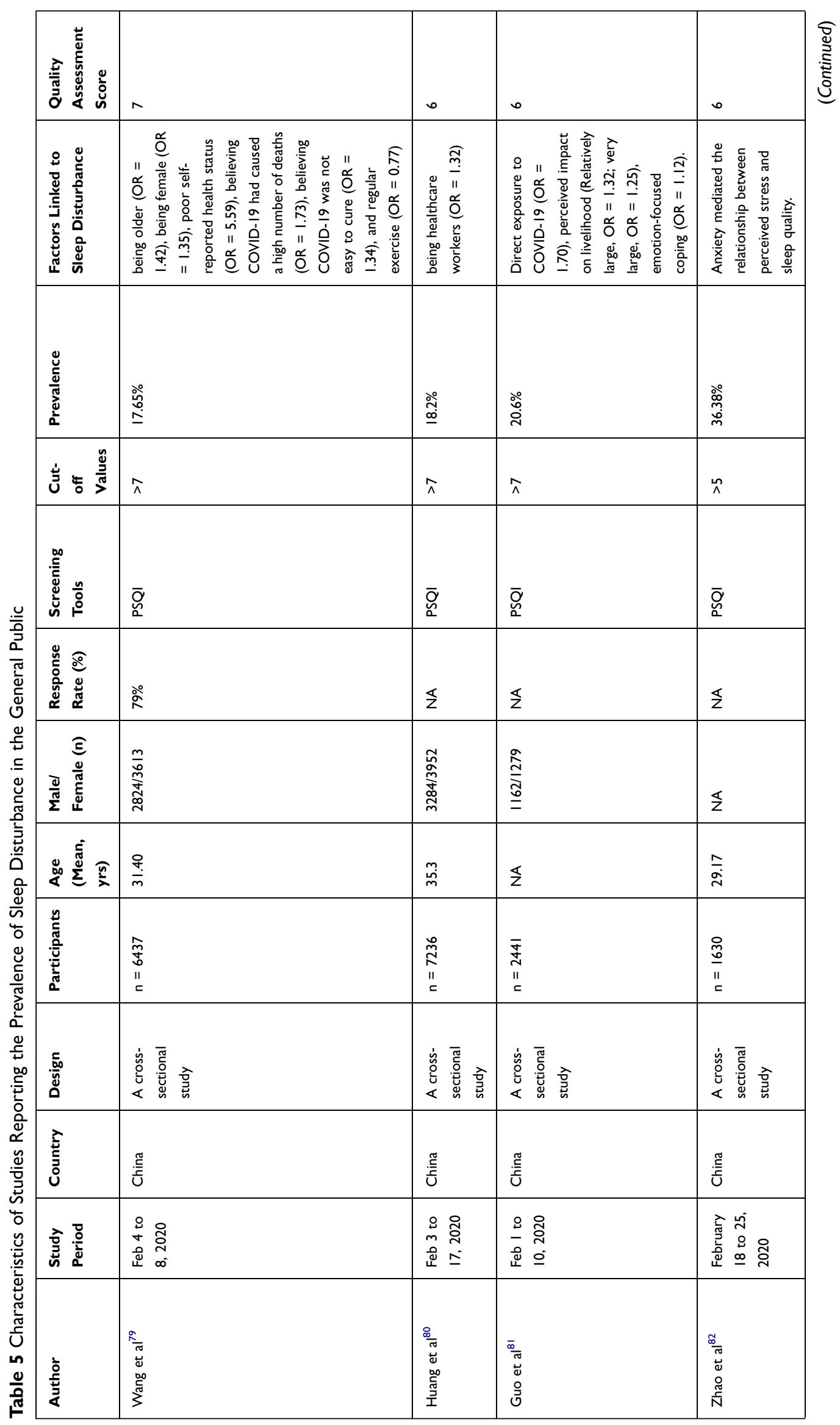




\begin{tabular}{|c|c|c|c|c|c|}
\hline 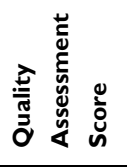 & 0 & 0 & 0 & 0 & in \\
\hline 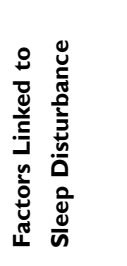 & 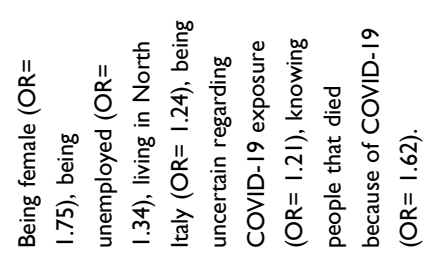 & 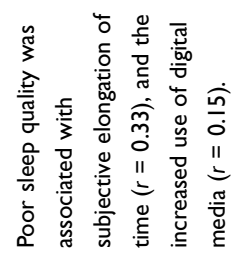 & $\mathbb{z}$ & 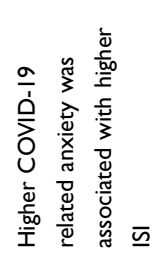 & 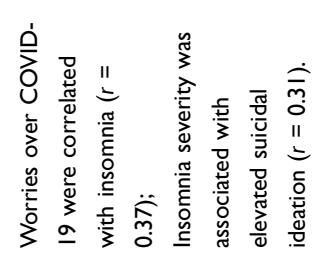 \\
\hline 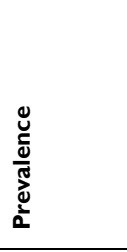 & $\stackrel{\circ}{\stackrel{\circ}{i}}$ & 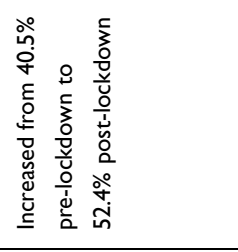 & 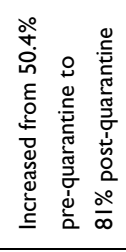 & 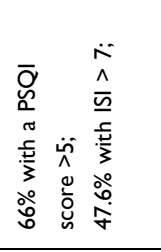 & ウ̊ \\
\hline 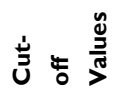 & $\stackrel{\sim}{\wedge}$ & $\stackrel{n}{\wedge}$ & $\stackrel{n}{\Lambda}$ & 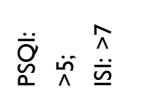 & $\stackrel{\infty}{\infty}$ \\
\hline 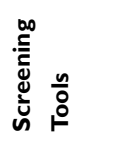 & $\bar{o}$ & $\overline{\mathrm{g}}$ & $\overline{0}$ & $\begin{array}{l}\ddot{\overline{\bar{g}}} \\
\underline{\underline{n}} \underline{\underline{\underline{n}}}\end{array}$ & $\underline{\overline{\mathrm{s}}}$ \\
\hline 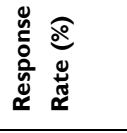 & $\mathbb{z}$ & $\mathbb{z}$ & 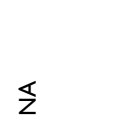 & $\mathbb{z}$ & $\mathbb{z}$ \\
\hline 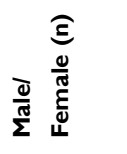 & 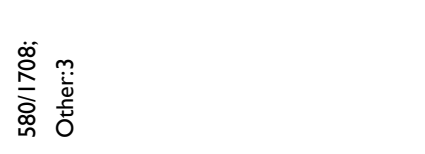 & 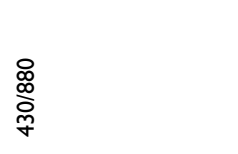 & $\stackrel{\infty}{\stackrel{\infty}{\gamma}}$ & $\overline{\stackrel{9}{q}}$ & 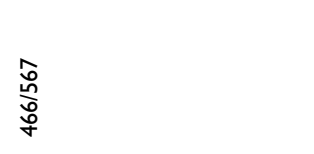 \\
\hline 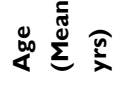 & : & $\begin{array}{l}\bar{\sigma} \\
\underset{\sim}{\sim}\end{array}$ & $\begin{array}{l}\stackrel{\sigma}{j} \\
\dot{f}\end{array}$ & $\stackrel{\infty}{\infty}$ & $\mathbb{Z}$ \\
\hline 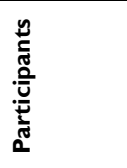 & 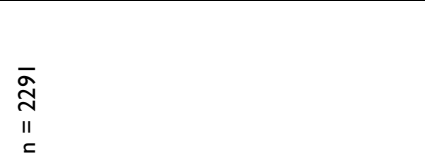 & $\begin{array}{l}\frac{0}{m} \\
\frac{11}{n} \\
=\end{array}$ & $\begin{array}{l}\overline{\underline{I}} \\
\bar{c} \\
\bar{c}\end{array}$ & $\begin{array}{l}\stackrel{m}{\underline{O}} \\
\text { "I }\end{array}$ & $\begin{array}{l}\frac{m}{0} \\
\frac{1}{11} \\
=\end{array}$ \\
\hline$\frac{\overline{.0}}{\bar{\Delta}}$ & 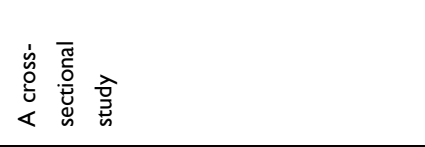 & 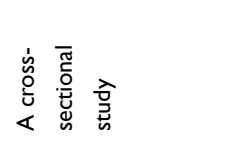 & 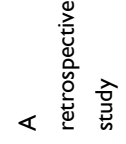 & 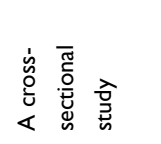 & 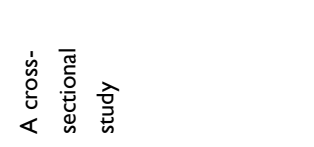 \\
\hline 离 & 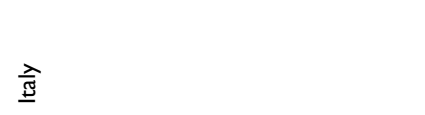 & 츠 & 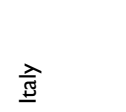 & 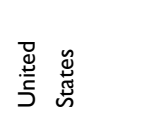 & 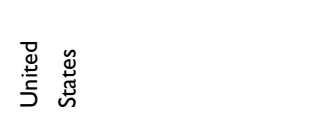 \\
\hline 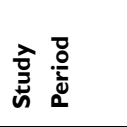 & 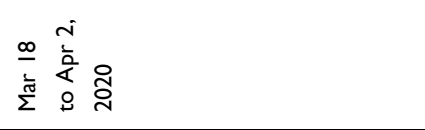 & 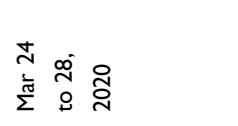 & 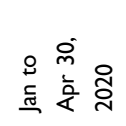 & 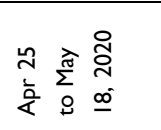 & 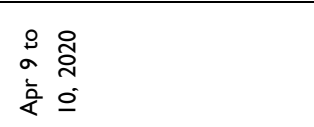 \\
\hline 旁 & 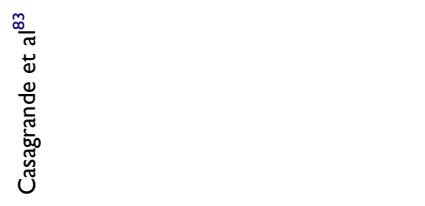 & 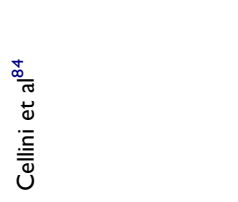 & 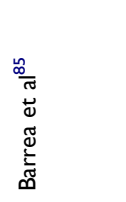 & 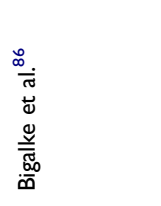 & 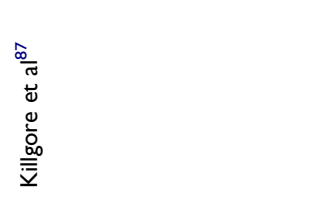 \\
\hline
\end{tabular}




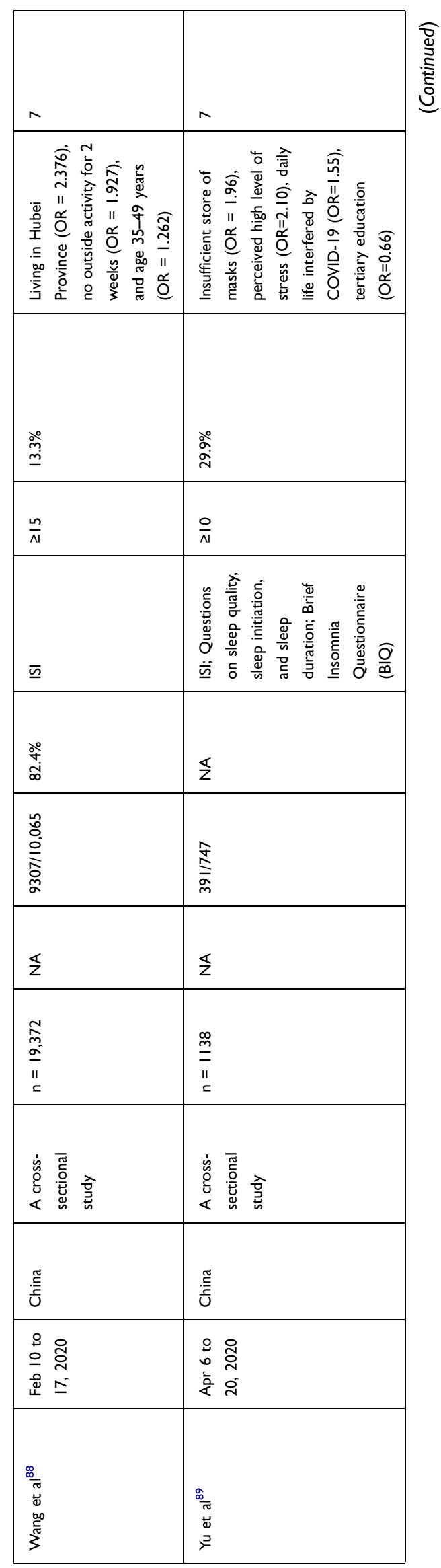




\begin{tabular}{|c|c|}
\hline 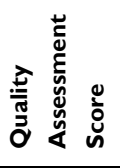 & $\infty$ \\
\hline 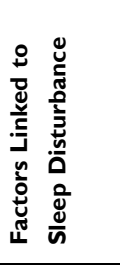 & 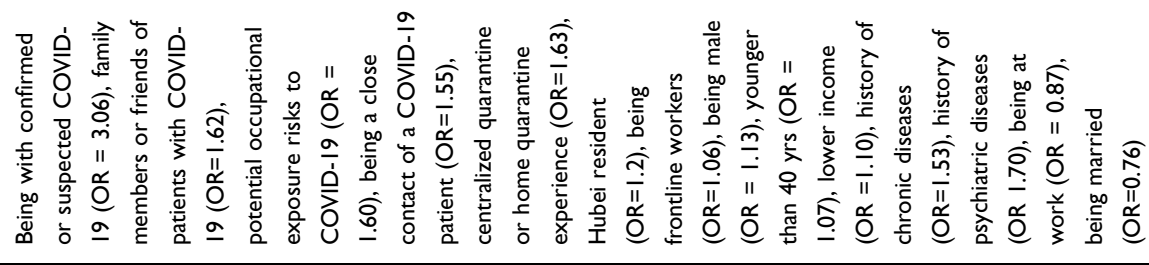 \\
\hline 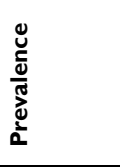 & خे \\
\hline 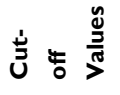 & $\stackrel{\infty}{\wedge}$ \\
\hline 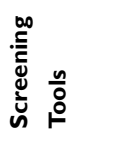 & $\underline{\bar{y}}$ \\
\hline 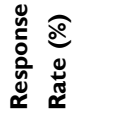 & $\begin{array}{l}\stackrel{\circ}{\circ} \\
\stackrel{2}{\circ}\end{array}$ \\
\hline 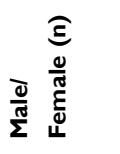 & $\begin{array}{l}\bar{\sigma} \\
\frac{\sigma}{\sigma} \\
\bar{N}\end{array}$ \\
\hline 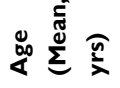 & $\begin{array}{l}\hat{a} \\
\hat{m}\end{array}$ \\
\hline 营 & $\begin{array}{l}0 \\
0 \\
0 \\
0.0 \\
11 \\
c\end{array}$ \\
\hline 递 & 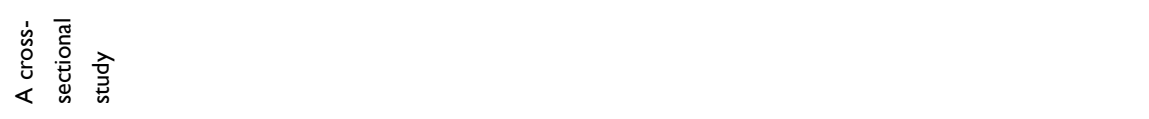 \\
\hline uे & 苞 \\
\hline 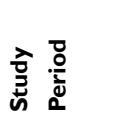 & 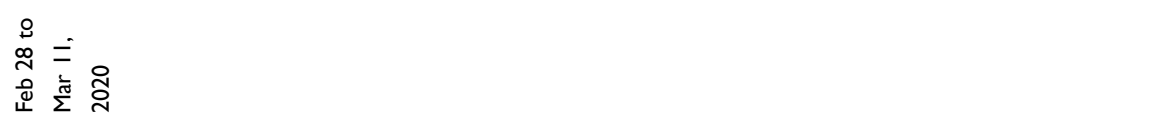 \\
\hline 六 & $\begin{array}{l}\frac{0}{\pi} \\
\frac{0}{0} \\
\dot{\bar{\omega}}\end{array}$ \\
\hline
\end{tabular}




\begin{tabular}{|c|c|c|c|c|c|}
\hline in & $\circ$ & $\circ$ & 。 & 。 & 。 \\
\hline 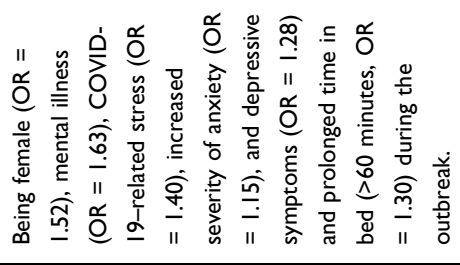 & z & $\Sigma$ & 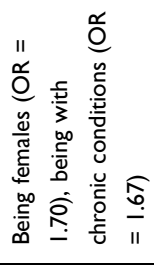 & 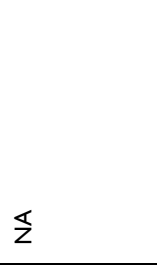 & 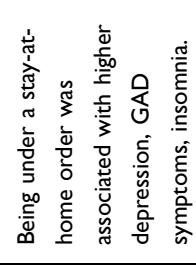 \\
\hline 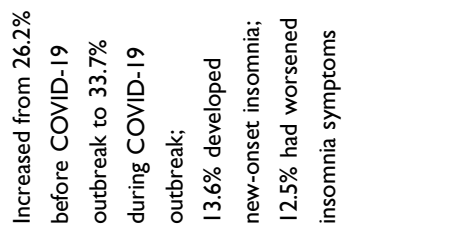 & $\begin{array}{l}\stackrel{\circ}{\circ} \\
\stackrel{+}{+}\end{array}$ & ڤั & 户े & $\stackrel{\circ}{\circ}$ & $\begin{array}{l}\stackrel{\circ}{\circ} \\
\stackrel{\infty}{\infty} \\
\text { m. }\end{array}$ \\
\hline$\lambda$ & ${ }_{\wedge}^{\infty}$ & $\frac{n}{\Lambda}$ & ${ }_{\wedge}^{\infty}$ & $\lambda$ & $\frac{\circ}{\wedge}$ \\
\hline$\underline{\bar{s}}$ & $\underline{\overline{ }}$ & $\underline{\overline{ }}$ & $\underline{\overline{ }}$ & $\underline{\overline{ }}$ & $\underline{\bar{g}}$ \\
\hline$\overleftarrow{z}$ & $\mathbb{Z}$ & $\mathbb{z}$ & $\mathbb{z}$ & $\mathbb{z}$ & $\mathbb{z}$ \\
\hline 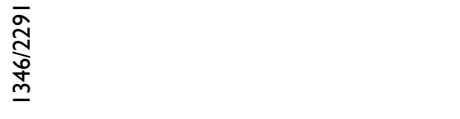 & $\begin{array}{l}\frac{1}{\infty} \\
\stackrel{0}{0} \\
0 \\
\end{array}$ & $\begin{array}{l}\frac{1}{\text { op }} \\
\text { ò } \\
\text { d. }\end{array}$ & $\frac{\hat{S}}{\bar{a}}$ & $\begin{array}{l}\widetilde{o} \\
\substack{q \\
\mathrm{~d}}\end{array}$ & 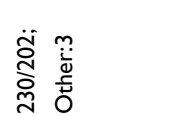 \\
\hline $\begin{array}{l}\stackrel{f}{+} \\
\dot{m}\end{array}$ & $\begin{array}{l}\stackrel{a}{\mathbf{d}} \\
\stackrel{\sim}{\infty}\end{array}$ & $\stackrel{\text { 어 }}{ }$ & $\mathcal{f}$ & 岂 & హ్ \\
\hline $\begin{array}{c}\hat{o} \\
\text { o } \\
\text { II } \\
c\end{array}$ & $\stackrel{N}{\underline{\underline{N}}}$ & 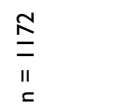 & $\frac{\frac{n}{2}}{\frac{n}{n}}$ & $\begin{array}{l}\bar{f} \\
11 \\
\varepsilon\end{array}$ & $\begin{array}{l}\stackrel{\mu}{\tilde{\gamma}} \\
\| 1 \\
\tilde{c}\end{array}$ \\
\hline 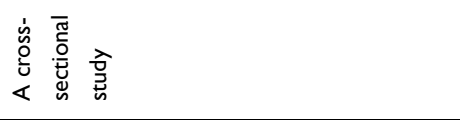 & 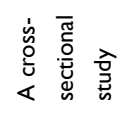 & 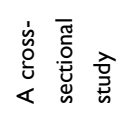 & 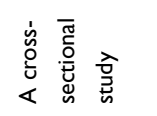 & 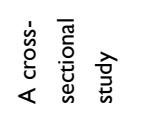 & 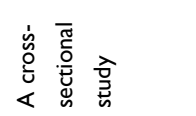 \\
\hline 䗆 & 胥 & 胥 & 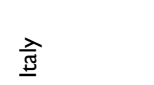 & $\begin{array}{l}\frac{7}{0} \\
\frac{5}{0} \\
\frac{0}{0}\end{array}$ & 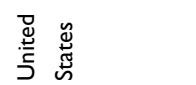 \\
\hline 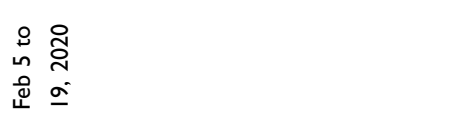 & 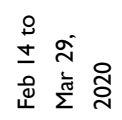 & 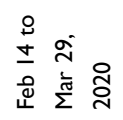 & 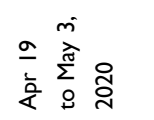 & 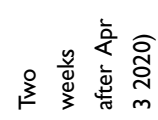 & 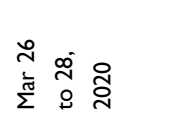 \\
\hline 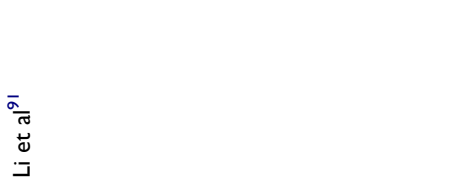 & 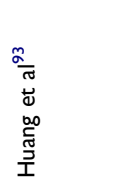 & 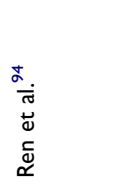 & 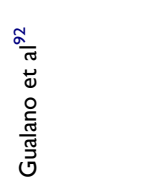 & 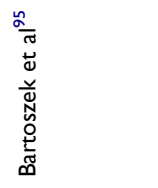 & 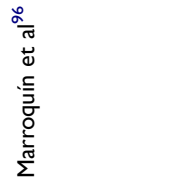 \\
\hline
\end{tabular}




\begin{tabular}{|c|c|c|}
\hline 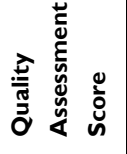 & in & 0 \\
\hline 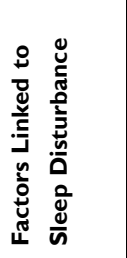 & 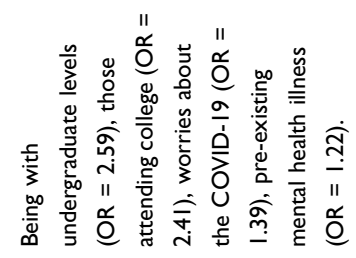 & 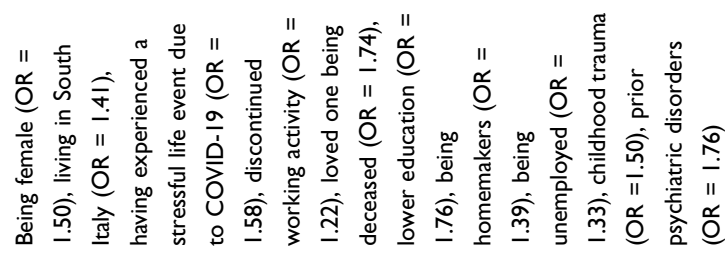 \\
\hline 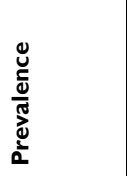 & $\frac{\partial 0}{\underline{\alpha}}$ & $\stackrel{\circ}{\stackrel{\circ}{n}}$ \\
\hline 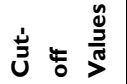 & $\frac{n}{N}$ & $\underset{N}{\mathbb{N}}$ \\
\hline 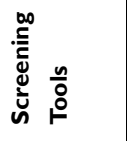 & $\underline{\bar{n}}$ & $\underline{\bar{\Xi}}$ \\
\hline 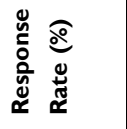 & $\overleftarrow{z}$ & $\mathbb{z}$ \\
\hline 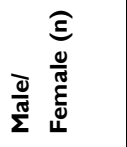 & 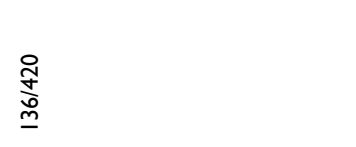 & 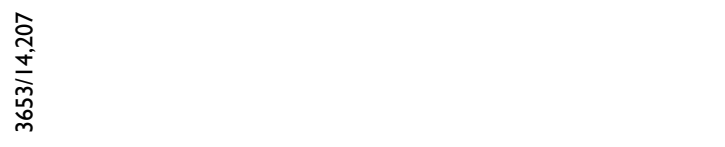 \\
\hline 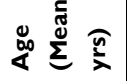 & 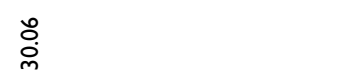 & 朵 \\
\hline 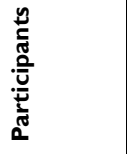 & $\begin{array}{l}\text { 员 } \\
\text { II } \\
=\end{array}$ & $\begin{array}{l}\frac{f}{a} \\
\frac{0}{11} \\
c\end{array}$ \\
\hline$\frac{.50}{\bar{D}}$ & 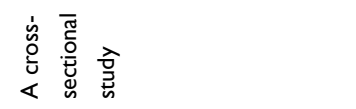 & 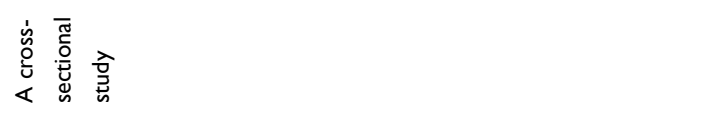 \\
\hline 童 & 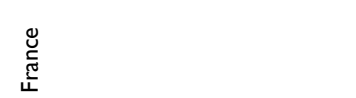 & 츨 \\
\hline 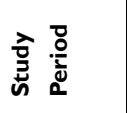 & 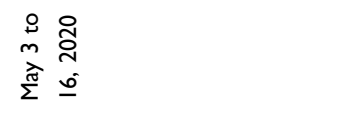 & 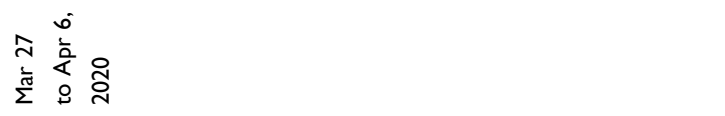 \\
\hline & 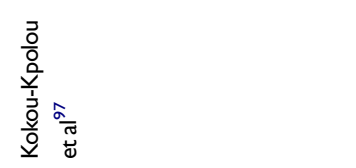 & 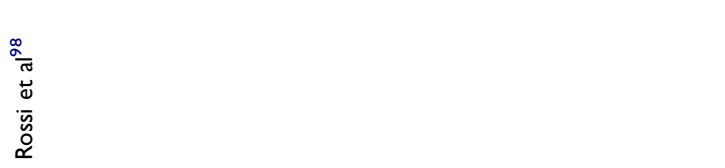 \\
\hline
\end{tabular}




\begin{tabular}{|c|c|c|c|c|}
\hline$\circ$ & $\circ$ & n & in & $\circ$ \\
\hline$\underline{z}$ & 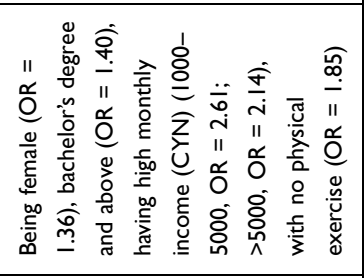 & 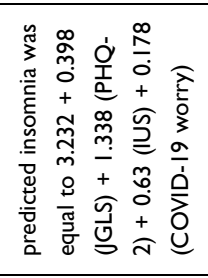 & 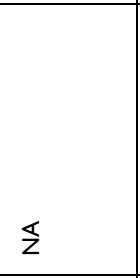 & 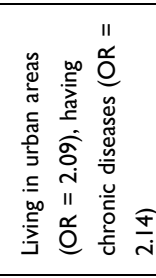 \\
\hline 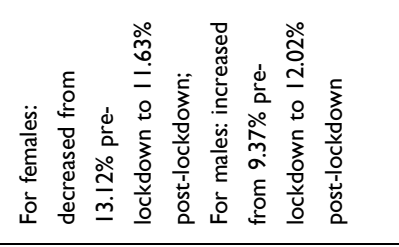 & 总 & $\stackrel{\circ}{\stackrel{\leftrightarrow}{\circ}}$ & $\begin{array}{c}\stackrel{\circ}{\circ} \\
\stackrel{m}{m}\end{array}$ & 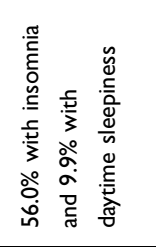 \\
\hline$\frac{t}{\lambda}$ & נin & $\underline{z}$ & $\frac{O}{N}$ & 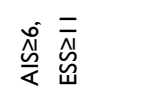 \\
\hline$\underline{\underline{\underline{ }}}$ & $\frac{n}{\alpha}$ & $\frac{n}{q}$ & $\frac{n}{4}$ & 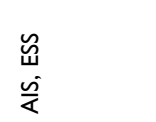 \\
\hline$\underline{z}$ & $\underline{z}$ & $\underline{z}$ & $\frac{\pi}{z}$ & $\frac{\pi}{z}$ \\
\hline 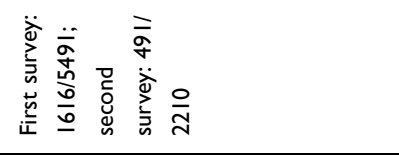 & $\begin{array}{l}0 \\
\text { o. } \\
0 \\
\infty \\
\end{array}$ & 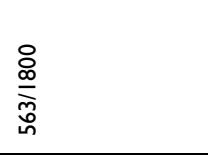 & 商 & 钫 \\
\hline 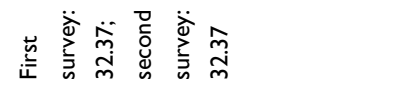 & $\underline{z}$ & $\underline{z}$ & 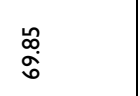 & $\underset{\dot{m}}{\vec{m}}$ \\
\hline 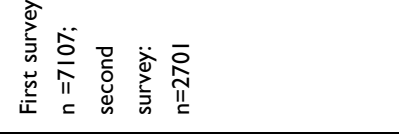 & 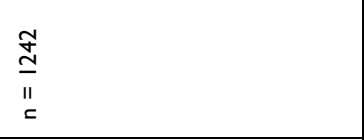 & 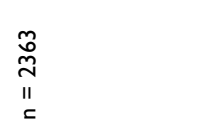 & $\begin{array}{l}\stackrel{m}{\underline{n}} \\
\underline{c}\end{array}$ & 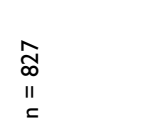 \\
\hline 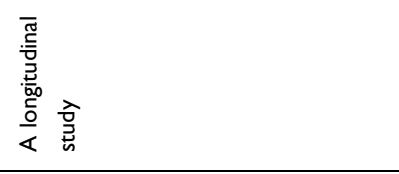 & 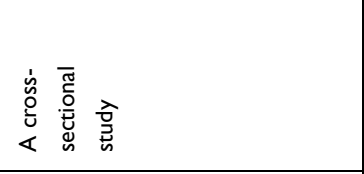 & 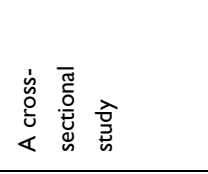 & 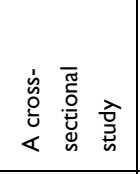 & 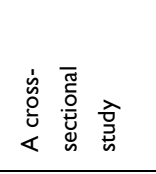 \\
\hline 촢 & 密 & 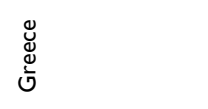 & 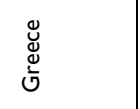 & $\begin{array}{l}\stackrel{8}{0} \\
\frac{0}{2} \\
\frac{0}{2}\end{array}$ \\
\hline 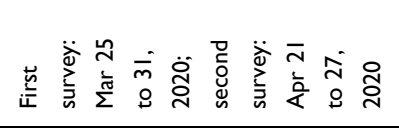 & 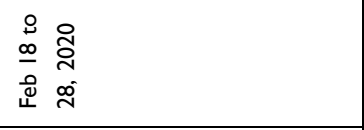 & 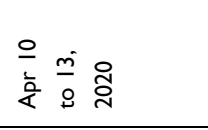 & $\S$ & 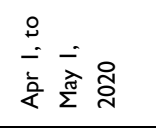 \\
\hline 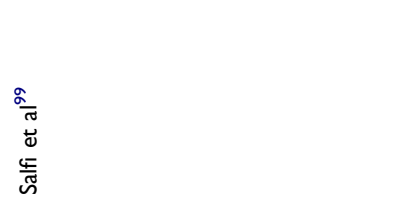 & 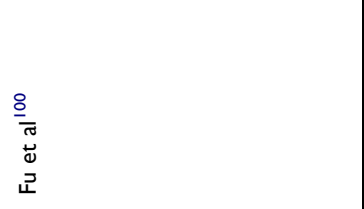 & 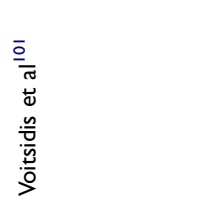 & 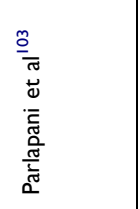 & 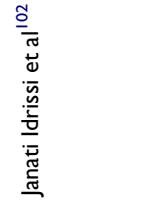 \\
\hline
\end{tabular}




\begin{tabular}{|c|c|c|}
\hline 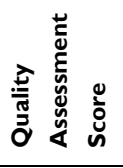 & in & $m$ \\
\hline 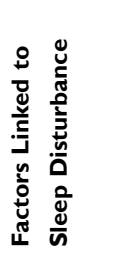 & 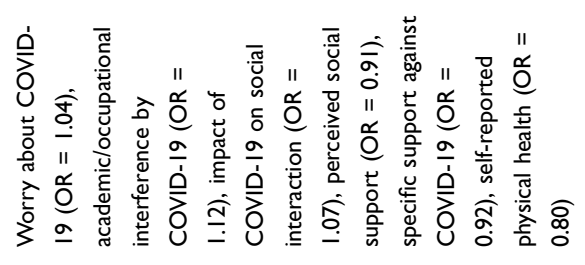 & 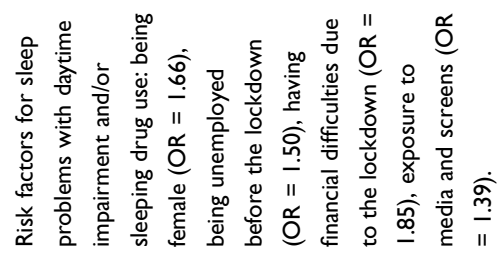 \\
\hline 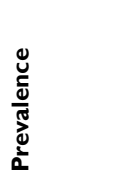 & 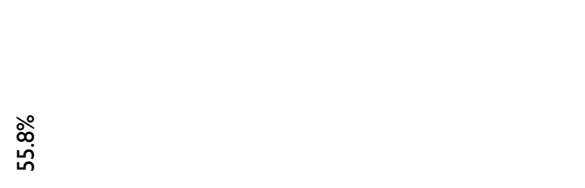 & $\stackrel{\circ}{\stackrel{\circ}{n}}$ \\
\hline 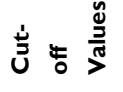 & i & $\S$ \\
\hline 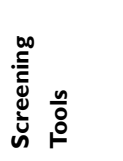 & 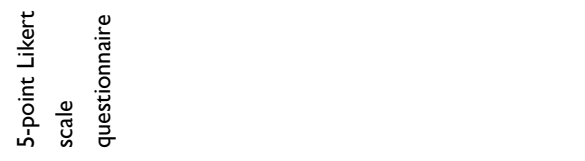 & 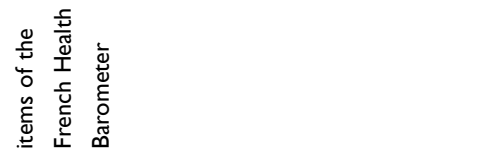 \\
\hline 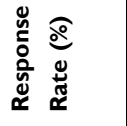 & $\S$ & $\S$ \\
\hline 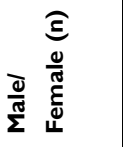 & 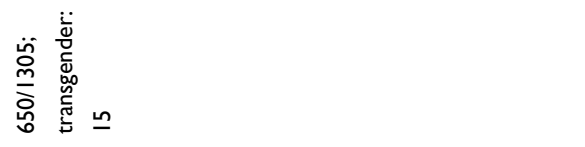 & $\S$ \\
\hline 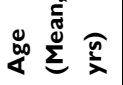 & $\begin{array}{l}\overline{\bar{\alpha}} \\
\stackrel{m}{m}\end{array}$ & $\S$ \\
\hline 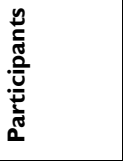 & $\begin{array}{l}\frac{8}{2} \\
\frac{1}{11} \\
c\end{array}$ & $\begin{array}{l}\underline{\underline{O}} \\
\frac{0}{11} \\
=\end{array}$ \\
\hline 点 & 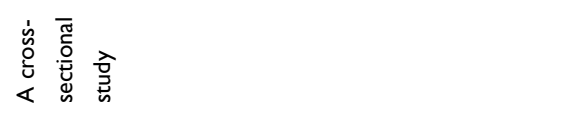 & 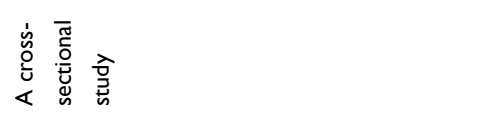 \\
\hline 章 & 莺 & 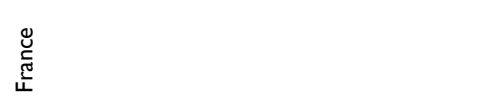 \\
\hline 窟 & 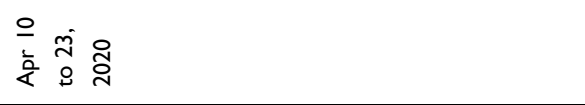 & 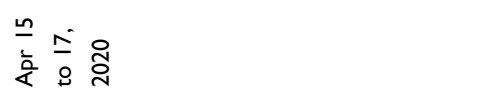 \\
\hline 童 & 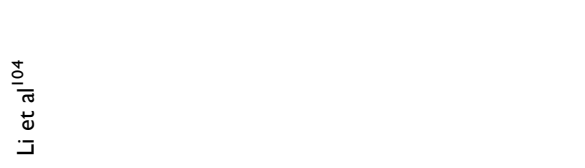 & 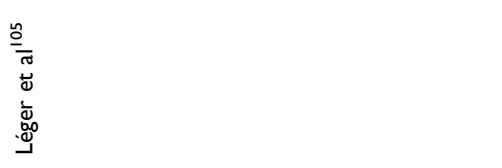 \\
\hline
\end{tabular}




\begin{tabular}{|c|c|c|c|}
\hline$\sigma$ & $\sigma$ & $\sigma$ & $\sigma$ \\
\hline 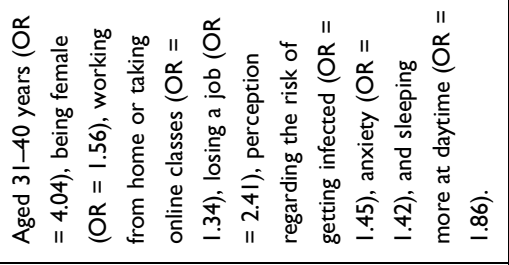 & 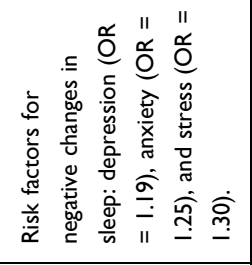 & $\stackrel{\nwarrow}{z}$ & 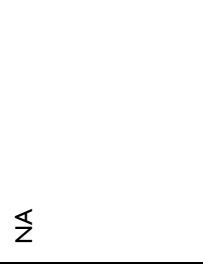 \\
\hline$\underset{\text { \ે }}{\stackrel{\circ}{\circ}}$ & 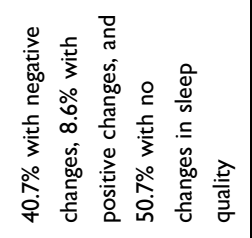 & 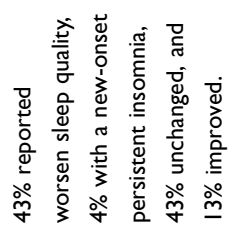 & $\begin{array}{l}\text { ळें } \\
\text { ஸे }\end{array}$ \\
\hline$\overleftarrow{z}$ & 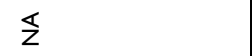 & 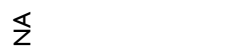 & $\overleftarrow{z}$ \\
\hline 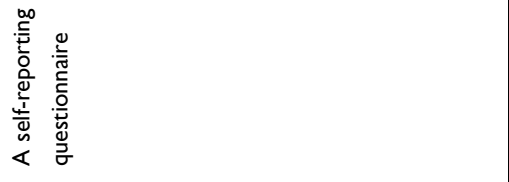 & 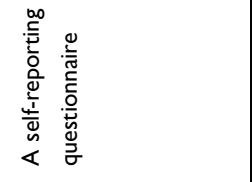 & 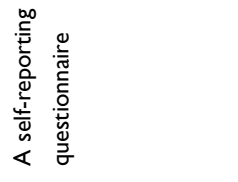 & 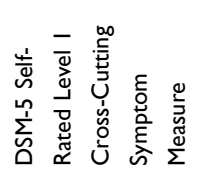 \\
\hline$\Sigma$ & $\mathbb{z}$ & $\mathbb{z}$ & $\mathbb{z}$ \\
\hline $\begin{array}{l}\text { ¿ั } \\
\text { ন্் }\end{array}$ & $\begin{array}{l}\frac{\sigma}{\sigma} \\
\frac{\sigma}{\sigma} \\
\sigma \\
\sigma\end{array}$ & $\frac{\circ}{\frac{1}{8}}$ & 总 \\
\hline 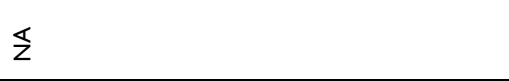 & 㓏 & $\mathbb{z}$ & $\begin{array}{l}\tilde{N} \\
\underset{j}{*}\end{array}$ \\
\hline 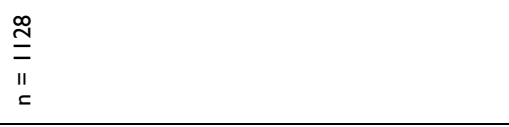 & $\begin{array}{l}\overline{\bar{g}} \\
\frac{g}{11} \\
c\end{array}$ & $\begin{array}{l}\stackrel{8}{g} \\
\text { II } \\
= \\
\end{array}$ & $\begin{array}{l}\stackrel{\circ}{\sigma} \\
11 \\
ᄃ \\
\end{array}$ \\
\hline 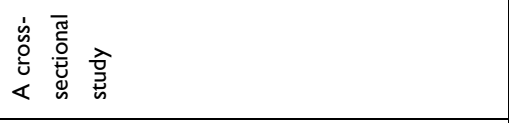 & 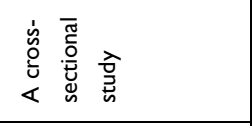 & 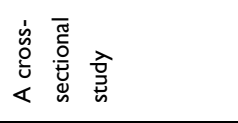 & 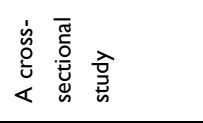 \\
\hline 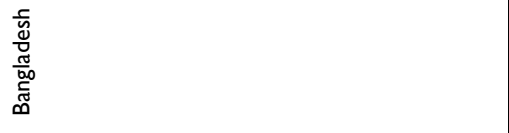 & 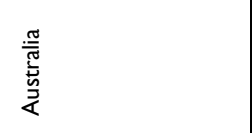 & 츨 & $\begin{array}{l}\overline{\bar{y}} \\
\bar{D}\end{array}$ \\
\hline 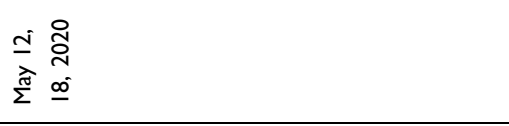 & 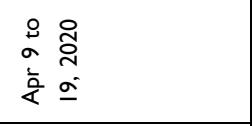 & 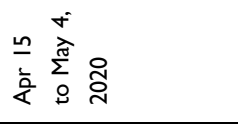 & 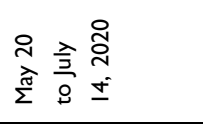 \\
\hline 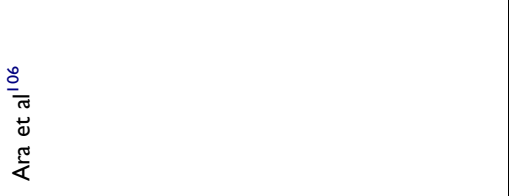 & 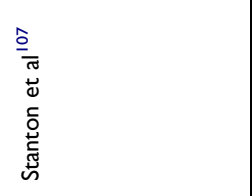 & 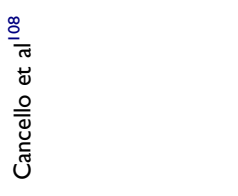 & 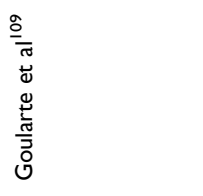 \\
\hline
\end{tabular}




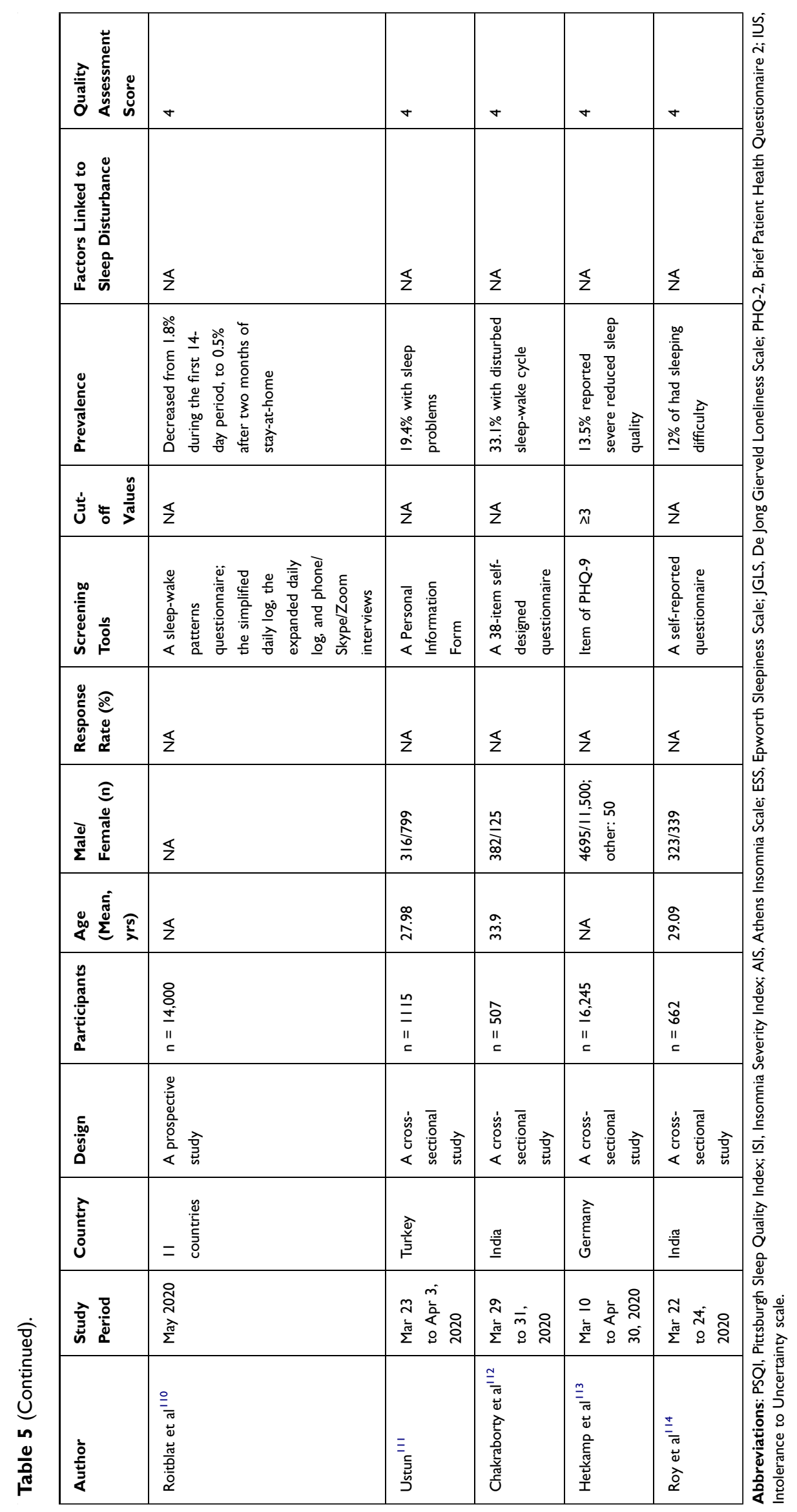


may have during COVID-19 lockdown. On the contrary, data from The Coconel Group showed an increase in the prevalence of sleep disturbance in young people aged 18-34 yrs when compared with older ones. ${ }^{18}$ Consistently, three cross-sectional studies reported an increase of sleep problems in college students from baseline to lockdown. ${ }^{123-125}$ It could be explained by the increased sleep vulnerability to stress caused by dramatic changes in their daily life and studies due to home confinement, school suspension, and reduced outdoor activity during the COVID-19 lockdown. Therefore, it could be speculated that the effects of age on sleep during COVID19 pandemic are complicated and inconclusive, and could be interfered by other social-psychological factors.

Sex difference has been reported in the prevalence of sleep problems. Females seemed to be more prone to have sleep problems than males when facing COVID-19 crisis. $^{79,83,91,92,98,100,105,106}$ However, a longitudinal study showed that although females generally scored higher in PSQI and ISI scores within the 4-week home confinement period, they reported a reduction in insomnia and other accompanied psychological symptoms, while males had an increase in PSQI and ISI scores, indicating a narrowed sex gap in sleep quality after a prolonged lockdown. ${ }^{99}$

Perceived COVID-19-related stress is another major contributor for sleep disturbance, possibly through a change in emotional state (eg, stress, depression and anxiety). ${ }^{82,86,89,91,101,107}$ Firstly, people who had direct or potential exposure to COVID-19 $9^{84,86,93}$ may be afraid of being infected and worry about being isolated and quarantined, all of which may exacerbate psychological distress and the accompanied symptoms of sleep disturbance. Secondly, post-traumatic stress due to the high mortality of COVID-19 and the unexpected death of someone close, ${ }^{83}$ along with the loneliness due to social distancing or quarantine $e^{90,98}$ increased psychological burden, making people prone to PTSD and sleep disturbance. Thirdly, negative attitude towards COVID-19, such as worries about COVID-19, perceived high death risk, perceived difficulty in treatment of COVID-19, being negative about COVID-19 control and emotion-focused coping style, may provoke cognitive arousal and disturb poor sleep quality. ${ }^{79,81,87,97,101,104,106}$ Fourthly, the more negative impacts do COVID-19 have on livelihood, the more likely do people develop sleep disturbance. ${ }^{81,83,89,98,104-106}$

Additionally, geographical factors (eg, living in epicenters or in urban areas), ${ }^{83,88,90,102}$ education experience, ${ }^{89,97,98,100}$ marital status, ${ }^{90}$ a history of chronic diseases or mental illnesses, ${ }^{57,79,90,92,97,98,102}$ and changes in daily life ${ }^{84,91,100,101,105,106}$ were also associated with sleep disturbance during COVID-19 pandemic.

Taken together, sleep disturbance in the general public, which is greatly influenced by social-psychological factors, could be compromised by reduced social jetlag and social sleep restriction during the pandemic. However, sleep disturbance-associated health consequences in the general public still need further investigation.

\section{Limitation}

There were several limitations in the review. Firstly, the majority of the included studies were cross-sectional design. More longitudinal studies are encouraged to determine the temporal changes of sleep disturbance during and after the pandemic. Secondly, the adoption of online surveys in most of the included studies limits the diagnosis of patterns of sleep disturbance and accurate assessment of disease severity. Thirdly, most of the included studies were descriptive. There is a lack of research to address the efficacy of targeted interventions including relaxation techniques, behavioral interventions, sleep medications by population on sleep disturbance and its associated health consequences. Fourthly, inclusion of studies in English only in the review may cause language bias.

\section{Conclusion}

In summary, COVID-19 exerts adverse impacts on sleep and brings great burden among various groups of populations. Sleep disturbance, mental illnesses, and physiologic illnesses form a vicious cycle to worsen the prognosis in COVID-19 patients. High workforce, shift work and COVID-19-related stress could increase the risk of sleep disturbance of HWs. For the general public, sleep quality seems more sensitive to social-psychological factors. Therefore, specific health strategies by population should be implemented to tackle sleep disturbance.

\section{Acknowledgment}

This work was funded by the National Natural Science Foundation of China (founding no. 81700084, 81770084, 81570082, 81700085), National Key R\&D Program of China (2018YFC1311900), and Shanghai Key Discipline for Respiratory Disease (2017ZZ02014). Ying Ni Lin, Zhuo Ran Liu and Shi Qi Li are co-first authors. 


\section{Disclosure}

The authors declare no conflict of interest.

\section{References}

1. Zielinski MR, McKenna JT, McCarley RW. Functions and mechanisms of sleep. AIMS Neurosci. 2016;3(1):67-104. doi:10.3934/Neuroscience.2016.1.67

2. Miller MA, Cappuccio FP. A systematic review of COVID-19 and obstructive sleep apnoea. Sleep Med Rev. 2021;55:101382. doi:10.1016/j.smrv.2020.101382

3. Salazar de Pablo G, Vaquerizo-Serrano J, Catalan A, et al. Impact of coronavirus syndromes on physical and mental health of health care workers: systematic review and meta-analysis. J Affect Disord. 2020;275:48-57. doi:10.1016/j.jad.2020.06.022

4. Alnofaiey YH, Alshehri HA, Alosaimi MM, et al. Sleep disturbances among physicians during COVID-19 pandemic. BMC Res Notes. 2020;13(1):493. doi:10.1186/s13104-020-05341-6

5. Loney PL, Chambers LW, Bennett KJ, Roberts JG, Stratford PW. Critical appraisal of the health research literature: prevalence or incidence of a health problem. Chronic Dis Can. 1998;19(4):170176.

6. Zhang H, Qin S, Zhang L, Feng Z, Fan C. A psychological investigation of coronavirus disease 2019 (COVID-19) patients in mobile cabin hospitals in Wuhan. Ann Transl Med. 2020;8 (15):941. doi:10.21037/atm-20-4907

7. Dai LL, Wang X, Jiang TC, et al. Anxiety and depressive symptoms among COVID-19 patients in Jianghan Fangcang Shelter Hospital in Wuhan, China. PLoS One. 2020;15(8):e0238416. doi:10.1371/journal.pone.0238416

8. Hao F, Tam W, Hu X, et al. A quantitative and qualitative study on the neuropsychiatric sequelae of acutely ill COVID-19 inpatients in isolation facilities. Transl Psychiatry. 2020;10(1):355. doi:10.1038/s41398-020-01039-2

9. Liguori C, Pierantozzi M, Spanetta M, et al. Subjective neurological symptoms frequently occur in patients with SARS-CoV2 infection. Brain Behav Immun. 2020;88:11-16. doi:10.1016/j. bbi.2020.05.037

10. Yue L, Wang J, Ju M, et al. How psychiatrists coordinate treatment for COVID-19: a retrospective study and experience from China. Gen Psychiatr. 2020;33(4):e100272. doi:10.1136/gpsych2020-100272

11. Iqbal Y, Al Abdulla MA, Albrahim S, Latoo J, Kumar R, Haddad PM. Psychiatric presentation of patients with acute SARS-CoV-2 infection: a retrospective review of 50 consecutive patients seen by a consultation-liaison psychiatry team. BJPsych Open. 2020;6 (5):e109. doi:10.1192/bjo.2020.85

12. Zhang J, Xu D, Xie B, et al. Poor-sleep is associated with slow recovery from lymphopenia and an increased need for ICU care in hospitalized patients with COVID-19: a retrospective cohort study. Brain Behav Immun. 2020;88:50-58. doi:10.1016/j. bbi.2020.05.075

13. Wu $\mathrm{C}, \mathrm{Hu} \mathrm{X}$, Song $\mathrm{J}$, et al. Mental health status and related influencing factors of COVID-19 survivors in Wuhan, China. Clin Transl Med. 2020;10(2):e52. doi:10.1002/ctm2.52

14. Mazza MG, De Lorenzo R, Conte C, et al. Anxiety and depression in COVID-19 survivors: role of inflammatory and clinical predictors. Brain Behav Immun. 2020;89:594-600. doi:10.1016/j. bbi.2020.07.037

15. Garrigues E, Janvier P, Kherabi Y, et al. Post-discharge persistent symptoms and health-related quality of life after hospitalization for COVID-19. J Infect. 2020;81(6):e4-e6. doi:10.1016/j. jinf.2020.08.029
16. Akter F, Mannan A, Mehedi HMH, et al. Clinical characteristics and short term outcomes after recovery from COVID-19 in patients with and without diabetes in Bangladesh. Diabetes Metab Syndr. 2020;14(6):2031-2038. doi:10.1016/j.dsx.2020.10.016

17. Meira ECM, Miyazawa M, Gozal D. Putative contributions of circadian clock and sleep in the context of SARS-CoV-2 infection. Eur Respir J. 2020;55:6.

18. Beck F, Léger D, Fressard L, Peretti-Watel P, Verger P. Covid-19 health crisis and lockdown associated with high level of sleep complaints and hypnotic uptake at the population level. J Sleep Res. 2020;30(1):e13119. doi:10.1111/jsr.13119

19. Kragholm K, Andersen MP, Gerds TA, et al.. Association between male sex and outcomes of Coronavirus disease 2019 (Covid-19) - a Danish nationwide, register-based study. Clin Infect Dis. 2020. doi:10.1093/cid/ciaa924

20. Bhatraju PK, Ghassemieh BJ, Nichols M, et al. Covid-19 in critically ill patients in the Seattle Region - Case Series. $N$ Engl J Med. 2020;382(21):2012-2022. doi:10.1056/NEJMoa2004500

21. Arentz M, Yim E, Klaff L, et al. Characteristics and outcomes of 21 critically ill patients with cOVID-19 in Washington State. JAMA. 2020;323(16):1612-1614. doi:10.1001/jama.2020.4326

22. Perger E, Soranna D, Pengo M, Meriggi P, Lombardi C, Parati G. Sleep disordered breathing among hospitalized patients due to COVID-19. Am J Respir Crit Care Med. 2020.

23. Gottlieb M, Sansom S, Frankenberger C, Ward E, Hota B. Clinical course and factors associated with hospitalization and critical illness among COVID-19 patients in Chicago, Illinois. Acad Emerg Med. 2020;27(10):963-973. doi:10.1111/acem.14104

24. Cariou B, Hadjadj S, Wargny M, et al. Phenotypic characteristics and prognosis of inpatients with COVID-19 and diabetes: the CORONADO study. Diabetologia. 2020;63(8):1500-1515. doi:10.1007/s00125-020-05180-x

25. McSharry D, Lam MT, Malhotra A. OSA as a probable risk factor for severe COVID-19. J Clin Sleep Med. 2020;16(9):1649. doi: $10.5664 /$ jcsm. 8708

26. McSharry D, Malhotra A. Potential influences of obstructive sleep apnea and obesity on COVID-19 severity. J Clin Sleep Med. 2020;16(9):1645. doi:10.5664/jcsm. 8538

27. Li YC, Bai WZ, Hashikawa T. The neuroinvasive potential of SARS$\mathrm{CoV} 2$ may play a role in the respiratory failure of COVID-19 patients. J Med Virol. 2020;92(6):552-555. doi:10.1002/jmv.25728

28. Najjar S, Najjar A, Chong DJ, et al. Central nervous system complications associated with SARS-CoV-2 infection: integrative concepts of pathophysiology and case reports. $J$ Neuroinflammation. 2020;17(1):231. doi:10.1186/s12974-02001896-0

29. Moldofsky H, Patcai J. Chronic widespread musculoskeletal pain, fatigue, depression and disordered sleep in chronic post-SARS syndrome; a case-controlled study. BMC Neurol. 2011;11(1):37. doi:10.1186/1471-2377-11-37

30. Vitti-Ruela BV, Dokkedal-Silva V, Rosa DS, Tufik S, Andersen ML. Possible sequelae in post-SARS-CoV-2 patients: effects on sleep and general health condition. Sleep Breath. 2020;1-2.

31. Culebras A. Cerebrovascular disease and sleep. Curr Neurol Neurosci Rep. 2004;4(2):164-169. doi:10.1007/s11910-0040032-6

32. Jiang Z, Zhu P, Wang L, et al. Psychological distress and sleep quality of COVID-19 patients in Wuhan, a lockdown city as the epicenter of COVID-19. J Psychiatr Res. 2020;136:595-602. doi:10.1016/j.jpsychires.2020.10.034

33. Yang X, Yang X, Kumar P, Cao B, Ma X, Li T. Social support and clinical improvement in COVID-19 positive patients in China. Nurs Outlook. 2020;68(6):830-837. doi:10.1016/j.outlook.2020.08.008 
34. Tan X, van Egmond L, Partinen M, Lange T, Benedict C. A narrative review of interventions for improving sleep and reducing circadian disruption in medical inpatients. Sleep Med. 2019;59:42-50. doi:10.1016/j.sleep.2018.08.007

35. Pisani MA, Friese RS, Gehlbach BK, Schwab RJ, Weinhouse GL, Jones SF. Sleep in the intensive care unit. Am J Respir Crit Care Med. 2015;191(7):731-738. doi:10.1164/rccm.201411-2099CI

36. Rogers JP, Chesney E, Oliver D, et al. Psychiatric and neuropsychiatric presentations associated with severe coronavirus infections: a systematic review and meta-analysis with comparison to the COVID-19 pandemic. Lancet Psychiatry. 2020;7(7):611-627. doi:10.1016/S2215-0366(20)30203-0

37. Wang S, Xie L, Xu Y, Yu S, Yao B, Xiang D. Sleep disturbances among medical workers during the outbreak of COVID-2019. Occup Med (Lond). 2020;70(5):364-369. doi:10.1093/occmed/ kqaa074

38. Tu ZH, He JW, Zhou N. Sleep quality and mood symptoms in conscripted frontline nurse in Wuhan, China during COVID-19 outbreak: a cross-sectional study. Medicine (Baltimore). 2020;99 (26):e20769. doi:10.1097/MD.0000000000020769

39. Cheng FF, Zhan SH, Xie AW, et al. Anxiety in Chinese pediatric medical staff during the outbreak of Coronavirus disease 2019: a cross-sectional study. Transl Pediatr. 2020;9(3):231-236. doi:10.21037/tp.2020.04.02

40. Qi J, Xu J, Li BZ, et al. The evaluation of sleep disturbances for Chinese frontline medical workers under the outbreak of COVID19. Sleep Med. 2020;72:1-4. doi:10.1016/j.sleep.2020.05.023

41. Zhou Y, Yang Y, Shi T, et al. Prevalence and demographic correlates of poor sleep quality among frontline health professionals in Liaoning Province, China During the COVID-19 outbreak. Front Psychiatry. 2020;11:520. doi:10.3389/ fpsyt.2020.00520

42. Wu K, Wei X. Analysis of psychological and sleep status and exercise rehabilitation of front-line clinical staff in the fight against COVID-19 in China. Med Sci Monit Basic Res. 2020;26:e924085. doi:10.12659/MSMBR.924085

43. Herrero San Martin A, Parra Serrano J, Diaz Cambriles T, et al. Sleep characteristics in health workers exposed to the COVID-19 pandemic. Sleep Med. 2020;75:388-394. doi:10.1016/j. sleep.2020.08.013

44. Zhao X, Zhang T, Li B, et al. Job-related factors associated with changes in sleep quality among healthcare workers screening for 2019 novel coronavirus infection: a longitudinal study. Sleep Med. 2020;75:21-26. doi:10.1016/j.sleep.2020.07.027

45. Wang W, Song W, Xia Z, et al. Sleep disturbance and psychological profiles of medical staff and non-medical staff during the early outbreak of COVID-19 in Hubei Province, China. Front Psychiatry. 2020;11:733. doi:10.3389/fpsyt.2020.00733

46. Jahrami H, BaHammam AS, AlGahtani H, et al. The examination of sleep quality for frontline healthcare workers during the outbreak of COVID-19. Sleep Breath. 2020;1-9.

47. Giardino DL, Huck-Iriart C, Riddick M, Garay A. The endless quarantine: the impact of the COVID-19 outbreak on healthcare workers after three months of mandatory social isolation in Argentina. Sleep Med. 2020;76:16-25. doi:10.1016/j. sleep.2020.09.022

48. Cai Z, Cui Q, Liu Z, et al. Nurses endured high risks of psychological problems under the epidemic of COVID-19 in a longitudinal study in Wuhan China. J Psychiatr Res. 2020;131:132137. doi:10.1016/j.jpsychires.2020.09.007

49. Zhang C, Yang L, Liu S, et al. Survey of insomnia and related social psychological factors among medical staff involved in the 2019 novel coronavirus disease outbreak. Front Psychiatry. 2020;11:306. doi:10.3389/fpsyt.2020.00306
50. Wang H, Huang D, Huang $\mathrm{H}$, et al. The psychological impact of COVID-19 pandemic on medical staff in Guangdong, China: a cross-sectional study. Psychol Med. 2020;1-9.

51. Lai J, Ma S, Wang Y, et al. Factors associated with mental health outcomes among health care workers exposed to Coronavirus disease 2019. JAMA Netw Open. 2020;3(3):e203976. doi:10.1001/jamanetworkopen.2020.3976

52. Que J, Shi L, Deng J, et al. Psychological impact of the COVID19 pandemic on healthcare workers: a cross-sectional study in China. Gen Psychiatr. 2020;33(3):e100259. doi:10.1136/gpsych2020-100259

53. Zhou Y, Wang W, Sun Y, et al. The prevalence and risk factors of psychological disturbances of frontline medical staff in china under the COVID-19 epidemic: workload should be concerned. $J$ Affect Disord. 2020;277:510-514. doi:10.1016/j. jad.2020.08.059

54. Zhang WR, Wang K, Yin L, et al. Mental health and psychosocial problems of medical health workers during the COVID-19 epidemic in China. Psychother Psychosom. 2020;89(4):242-250. doi:10.1159/000507639

55. Liang Y, Wu K, Zhou Y, Huang X, Zhou Y, Liu Z. Mental health in frontline medical workers during the 2019 novel coronavirus disease epidemic in China: a comparison with the general population. Int $J$ Environ Res Public Health. 2020;17(18):18. doi:10.3390/ijerph17186550

56. Zhang H, Shi Y, Jing P, Zhan P, Fang Y, Wang F. Posttraumatic stress disorder symptoms in healthcare workers after the peak of the COVID-19 outbreak: a survey of a large tertiary care hospital in Wuhan. Psychiatry Res. 2020;294:113541. doi:10.1016/j. psychres.2020.113541

57. Florin M, Pinar U, Chavigny E, et al. Socio-economic and psychological impact of the COVID-19 outbreak on private practice and public hospital radiologists. Eur J Radiol. 2020;132:109285. doi:10.1016/j.ejrad.2020.109285

58. Jain A, Singariya G, Kamal M, Kumar M, Jain A, Solanki RK. COVID-19 pandemic: psychological impact on anaesthesiologists. Indian J Anaesth. 2020;64(9):774-783. doi:10.4103/ija.IJA 69720

59. Almater AI, Tobaigy MF, Younis AS, Alaqeel MK, Abouammoh MA. Effect of 2019 coronavirus pandemic on ophthalmologists practicing in Saudi Arabia: a psychological health assessment. Middle East Afr J Ophthalmol. 2020;27(2):79-85. doi:10.4103/ meajo.MEAJO 22020

60. Cai Q, Feng H, Huang J, et al. The mental health of frontline and non-frontline medical workers during the coronavirus disease 2019 (COVID-19) outbreak in China: a case-control study. $J$ Affect Disord. 2020;275:210-215. doi:10.1016/j.jad.2020.06.031

61. Khanal P, Devkota N, Dahal M, Paudel K, Joshi D. Mental health impacts among health workers during COVID-19 in a low resource setting: a cross-sectional survey from Nepal. Global Health. 2020;16(1):89. doi:10.1186/s12992-020-00621-z

62. Şahin MK, Aker S, Şahin G, Karabekiroğlu A. Prevalence of depression, anxiety, distress and insomnia and related factors in healthcare workers during COVID-19 pandemic in Turkey. J Community Health. 2020;45(6):1168-1177. doi:10.1007/s10900-020-00921-w

63. Alshekaili M, Hassan W, Al Said N, et al. Factors associated with mental health outcomes across healthcare settings in Oman during COVID-19: frontline versus non-frontline healthcare workers. BMJ Open. 2020;10(10):e042030. doi:10.1136/bmjopen-2020042030

64. Rossi R, Socci V, Pacitti F, et al. Mental health outcomes among frontline and second-line health care workers during the Coronavirus disease 2019 (COVID-19) pandemic in Italy. JAMA Netw Open. 2020;3(5):e2010185. doi:10.1001/jamanetworkopen. 2020.10185 
65. Zhan Y, Liu Y, Liu H, et al. Factors associated with insomnia among Chinese front-line nurses fighting against COVID-19 in Wuhan: a cross-sectional survey. J Nurs Manag. 2020;28 (7):1525-1535. doi:10.1111/jonm.13094

66. Abdulah DM, Musa DH. Insomnia and stress of physicians during COVID-19 outbreak. Sleep Medicine: X. 2020;2:100017. doi:10.1016/j.sleepx.2020.100017

67. Mosheva M, Hertz-Palmor N, Dorman Ilan S, et al. Anxiety, pandemic-related stress and resilience among physicians during the COVID-19 pandemic. Depress Anxiety. 2020;37(10):965-971. doi:10.1002/da.23085

68. Sharif S, Amin F, Hafiz M, et al. COVID 19-depression and neurosurgeons. World Neurosurg. 2020;140:e401-e410. doi:10.1016/j.wneu.2020.06.007

69. Bhargava S, Sarkar R, Kroumpouzos G. Mental distress in dermatologists during COVID-19 pandemic: assessment and risk factors in a global, cross-sectional study. Dermatol Ther. 2020;33(6):e14161.

70. Qiu D, Yu Y, Li RQ, Li YL, Xiao SY. Prevalence of sleep disturbances in Chinese healthcare professionals: a systematic review and meta-analysis. Sleep Med. 2020;67:258-266. doi:10.1016/j.sleep.2019.01.047

71. Zhuo K, Gao C, Wang X, Zhang C, Wang Z. Stress and sleep: a survey based on wearable sleep trackers among medical and nursing staff in Wuhan during the COVID-19 pandemic. Gen Psychiatr. 2020;33(3):e100260.

72. Diomidous M. Sleep and motion disorders of physicians and nurses working in hospitals facing the pandemic of COVID 19. Med Arch. 2020;74(3):210-215. doi:10.5455/ medarh.2020.74.210-215

73. Ballesio A, Lombardo C, Lucidi F, Violani C. Caring for the carers: advice for dealing with sleep problems of hospital staff during the COVID-19 outbreak. J Sleep Res. 2020;30(1):e13096. doi:10.1111/jsr.13096

74. Lim RK, Wambier CG, Goren A. Are night shift workers at an increased risk for COVID-19? Med Hypotheses. 2020;144:110147. doi:10.1016/j.mehy.2020.110147

75. Rizza S, Coppeta L, Grelli S, et al. High body mass index and night shift work are associated with COVID-19 in health care workers. J Endocrinol Invest. 2020;1-5.

76. Zhang $\mathrm{S}, \mathrm{Xu} \mathrm{Y,} \mathrm{Wu} \mathrm{K}$, et al. Improved night shift schedule related to the mortality of critically ill patients with Corona Virus disease 2019. Sleep Med. 2020;75:354-360. doi:10.1016/j. sleep. 2020.08 .010

77. Liu H, Liu J, Chen M, et al. Sleep problems of healthcare workers in tertiary hospital and influencing factors identified through a multilevel analysis: a cross-sectional study in China. BMJ Open. 2019;9(12):e032239. doi:10.1136/bmjopen-2019-032239

78. Phua DH, Tang HK, Tham KY. Coping responses of emergency physicians and nurses to the 2003 severe acute respiratory syndrome outbreak. Acad Emerg Med. 2005;12(4):322-328. doi:10.1197/j.aem.2004.11.015

79. Wang J, Gong Y, Chen Z, et al. Sleep disturbances among Chinese residents during the Coronavirus disease 2019 outbreak and associated factors. Sleep Med. 2020;74:199-203. doi:10.1016/j.sleep.2020.08.002

80. Huang Y, Zhao N. Generalized anxiety disorder, depressive symptoms and sleep quality during COVID-19 outbreak in China: a web-based cross-sectional survey. Psychiatry Res. 2020;288:112954. doi:10.1016/j.psychres.2020.112954

81. Guo J, Feng XL, Wang XH, van IJzendoorn MH. Coping with COVID-19: exposure to COVID-19 and negative impact on livelihood predict elevated mental health problems in Chinese adults. Int J Environ Res Public Health. 2020;17(11):11. doi:10.3390/ ijerph17113857
82. Zhao X, Lan M, Li H, Yang J. Perceived stress and sleep quality among the non-diseased general public in China during the 2019 coronavirus disease: a moderated mediation model. Sleep Med. 2020.

83. Casagrande M, Favieri F, Tambelli R, Forte G. The enemy who sealed the world: effects quarantine due to the COVID-19 on sleep quality, anxiety, and psychological distress in the Italian population. Sleep Med. 2020;75:12-20. doi:10.1016/j. sleep.2020.05.011

84. Cellini N, Canale N, Mioni G, Costa S. Changes in sleep pattern, sense of time and digital media use during COVID-19 lockdown in Italy. J Sleep Res. 2020;29(4):e13074. doi:10.1111/jsr.13074

85. Barrea L, Pugliese G, Framondi L, et al. Does Sars-Cov-2 threaten our dreams? Effect of quarantine on sleep quality and body mass index. J Transl Med. 2020;18(1):318. doi:10.1186/s12967020-02465-y

86. Bigalke JA, Greenlund IM, Carter JR. Sex differences in selfreport anxiety and sleep quality during COVID-19 stay-at-home orders. Biol Sex Differ. 2020;11(1):56. doi:10.1186/s13293-02000333-4

87. Killgore WDS, Cloonan SA, Taylor EC, Fernandez F, Grandner MA, Dailey NS. Suicidal ideation during the COVID-19 pandemic: the role of insomnia. Psychiatry Res. 2020;290:113134. doi:10.1016/j.psychres.2020.113134

88. Wang S, Zhang Y, Ding W, et al. Psychological distress and sleep problems when people are under interpersonal isolation during an epidemic: a nationwide multicenter cross-sectional study. Eur Psychiatry. 2020;63(1):e77. doi:10.1192/j.eurpsy.2020.78

89. Yu BY, Yeung WF, Lam JC, et al. Prevalence of sleep disturbances during COVID-19 outbreak in an urban Chinese population: a cross-sectional study. Sleep Med. 2020;74:18-24. doi:10.1016/j.sleep.2020.07.009

90. Shi L, Lu ZA, Que JY, et al. Prevalence of and risk factors associated with mental health symptoms among the general population in china during the Coronavirus disease 2019 pandemic. JAMA Netw Open. 2020;3(7):e2014053. doi:10.1001/ jamanetworkopen.2020.14053

91. Li Y, Qin Q, Sun Q, Sanford LD, Vgontzas AN, Tang X. Insomnia and psychological reactions during the COVID-19 outbreak in China. J Clin Sleep Med. 2020;16(8):1417-1418.

92. Gualano MR, Lo Moro G, Voglino G, Bert F, Siliquini R. Effects of Covid-19 lockdown on mental health and sleep disturbances in Italy. Int $J$ Environ Res Public Health. 2020;17(13):13. doi:10.3390/ijerph17134779

93. Huang Y, Wang Y, Zeng L, et al. Prevalence and correlation of anxiety, insomnia and somatic symptoms in a Chinese population during the COVID-19 epidemic. Front Psychiatry. 2020;11:568329. doi:10.3389/fpsyt.2020.568329

94. Ren Y, Qian W, Li Z, et al. Public mental health under the longterm influence of COVID-19 in China: geographical and temporal distribution. J Affect Disord. 2020;277:893-900. doi:10.1016/j. jad.2020.08.045

95. Bartoszek A, Walkowiak D, Bartoszek A, Kardas G. Mental wellbeing (Depression, Loneliness, Insomnia, Daily Life Fatigue) during COVID-19 related home-confinement-A Study from Poland. Int $J$ Environ Res Public Health. 2020;17(20):20. doi:10.3390/ijerph17207417

96. Marroquín B, Vine V, Morgan R. Mental health during the COVID-19 pandemic: effects of stay-at-home policies, social distancing behavior, and social resources. Psychiatry Res. 2020;293:113419. doi:10.1016/j.psychres.2020.113419

97. Kokou-Kpolou CK, Megalakaki O, Laimou D, Kousouri M. Insomnia during COVID-19 pandemic and lockdown: prevalence, severity, and associated risk factors in French population. Psychiatry Res. 2020;290:113128. doi:10.1016/j. psychres.2020.113128 
98. Rossi R, Socci V, Talevi D, et al. COVID-19 pandemic and lockdown measures impact on mental health among the general population in Italy. Front Psychiatry. 2020;11:790. doi:10.3389/ fpsyt.2020.00790

99. Salfi F, Lauriola M, Amicucci G, et al. Gender-related time course of sleep disturbances and psychological symptoms during the COVID-19 lockdown: a longitudinal study on the Italian population. Neurobiol Stress. 2020;13:100259. doi:10.1016/j. ynstr.2020.100259

100. Fu W, Wang C, Zou L, et al. Psychological health, sleep quality, and coping styles to stress facing the COVID-19 in Wuhan, China. Transl Psychiatry. 2020;10(1):225. doi:10.1038/s41398020-00913-3

101. Voitsidis P, Gliatas I, Bairachtari V, et al. Insomnia during the COVID-19 pandemic in a Greek population. Psychiatry Res. 2020;289:113076. doi:10.1016/j.psychres.2020.113076

102. Janati Idrissi A, Lamkaddem A, Benouajjit A, et al. Sleep quality and mental health in the context of COVID-19 pandemic and lockdown in Morocco. Sleep Med. 2020;74:248-253. doi:10.1016/j.sleep.2020.07.045

103. Parlapani E, Holeva V, Nikopoulou VA, et al. Intolerance of uncertainty and loneliness in older adults during the COVID-19 Pandemic. Front Psychiatry. 2020;11:842. doi:10.3389/ fpsyt.2020.00842

104. Li DJ, Ko NY, Chen YL, et al. COVID-19-related factors associated with sleep disturbance and suicidal thoughts among the Taiwanese public: a Facebook Survey. Int J Environ Res Public Health. 2020;17:12.

105. Léger D, Beck F, Fressard L, Verger P, Peretti-Watel P. Poor sleep associated with overuse of media during the COVID-19 lockdown. Sleep. 2020;43(10):10. doi:10.1093/sleep/zsaa125

106. Ara T, Rahman MM, Hossain MA, Ahmed A. Identifying the associated risk factors of sleep disturbance during the COVID-19 lockdown in Bangladesh: a web-based survey. Front Psychiatry. 2020;11:580268. doi:10.3389/fpsyt.2020.580268

107. Stanton R, To QG, Khalesi S, et al. Depression, anxiety and stress during COVID-19: associations with changes in physical activity, sleep, tobacco and alcohol use in Australian adults. Int J Environ Res Public Health. 2020;17(11):11. doi:10.3390/ijerph17114065

108. Cancello R, Soranna D, Zambra G, Zambon A, Invitti C. Determinants of the lifestyle changes during COVID-19 pandemic in the residents of Northern Italy. Int $J$ Environ Res Public Health. 2020;17(17):17. doi:10.3390/ijerph17176287

109. Goularte JF, Serafim SD, Colombo R, Hogg B, Caldieraro MA, Rosa AR. COVID-19 and mental health in Brazil: psychiatric symptoms in the general population. $J$ Psychiatr Res. 2021;132:32-37. doi:10.1016/j.jpsychires.2020.09.021

110. Roitblat Y, Burger J, Leit A, et al. Stay-at-home circumstances do not produce sleep disorders: an international survey during the COVID-19 pandemic. J Psychosom Res. 2020;139:110282. doi:10.1016/j.jpsychores.2020.110282

111. Ustun G. Determining depression and related factors in a society affected by COVID-19 pandemic. Int J Soc Psychiatry. 2020;20764020938807. doi:10.1177/0020764020938807

112. Chakraborty K, Chatterjee M. Psychological impact of COVID19 pandemic on general population in West Bengal: a crosssectional study. Indian $J$ Psychiatry. 2020;62(3):266-272. doi:10.4103/psychiatry.IndianJPsychiatry_276_20
113. Hetkamp M, Schweda A, Bäuerle A, et al. Sleep disturbances, fear, and generalized anxiety during the COVID-19 shut down phase in Germany: relation to infection rates, deaths, and German stock index DAX. Sleep Med. 2020;75:350-353. doi:10.1016/j. sleep.2020.08.033

114. Roy D, Tripathy S, Kar SK, Sharma N, Verma SK, Kaushal V. Study of knowledge, attitude, anxiety \& perceived mental healthcare need in Indian population during COVID-19 pandemic. Asian J Psychiatr. 2020;51:102083. doi:10.1016/j. ajp. 2020.102083

115. Cao XL, Wang SB, Zhong BL, et al. The prevalence of insomnia in the general population in China: a meta-analysis. PLoS One. 2017;12(2):e0170772. doi:10.1371/journal.pone.0170772

116. Gao C, Scullin MK. Sleep health early in the coronavirus disease 2019 (COVID-19) outbreak in the United States: integrating longitudinal, cross-sectional, and retrospective recall data. Sleep Med. 2020;73:1-10. doi:10.1016/j.sleep.2020.06.032

117. Kocevska D, Blanken TF, Van Someren EJW, Rösler L. Sleep quality during the COVID-19 pandemic: not one size fits all. Sleep Med. 2020;76:86-88. doi:10.1016/j.sleep.2020.09.029

118. Blume C, Schmidt MH, Cajochen C. Effects of the COVID-19 lockdown on human sleep and rest-activity rhythms. Curr Biol. 2020;30(14):R795-r797. doi:10.1016/j.cub.2020.06.021

119. Leone MJ, Sigman M, Golombek DA. Effects of lockdown on human sleep and chronotype during the COVID-19 pandemic. Curr Biol. 2020;30(16):R930-r931. doi:10.1016/j. cub.2020.07.015

120. Au J, Reece J. The relationship between chronotype and depressive symptoms: a meta-analysis. J Affect Disord. 2017;218:93104. doi:10.1016/j.jad.2017.04.021

121. Czeisler CA, Dumont M, Duffy JF, et al. Association of sleepwake habits in older people with changes in output of circadian pacemaker. Lancet. 1992;340(8825):933-936. doi:10.1016/01406736(92)92817-Y

122. Kim HJ, Kim REY, Kim S, et al. Sex differences in deterioration of sleep properties associated with aging: a 12-year longitudinal cohort study. J Clin Sleep Med. 2021;17(5):964-972. doi:10.5664/jesm.9072

123. Marelli S, Castelnuovo A, Somma A, et al. Impact of COVID-19 lockdown on sleep quality in university students and administration staff. J Neurol. 2021;268(1):8-15. doi:10.1007/s00415-02010056-6

124. Romero-Blanco C, Rodríguez-Almagro J, Onieva-Zafra MD, Parra-Fernández ML, Prado-Laguna MDC, Hernández-Martínez A. Sleep pattern changes in nursing students during the COVID19 lockdown. Int J Environ Res Public Health. 2020;17(14):14. doi:10.3390/ijerph17145222

125. Kaparounaki CK, Patsali ME, Mousa DV, Papadopoulou EVK, Papadopoulou KKK, Fountoulakis KN. University students' mental health amidst the COVID-19 quarantine in Greece. Psychiatry Res. 2020;290:113111. doi:10.1016/j.psychres.2020.113111 


\section{Publish your work in this journal}

Nature and Science of Sleep is an international, peer-reviewed, open access journal covering all aspects of sleep science and sleep medicine, including the neurophysiology and functions of sleep, the genetics of sleep, sleep and society, biological rhythms, dreaming, sleep disorders and therapy, and strategies to optimize healthy sleep.

Submit your manuscript here: https://www.dovepress.com/nature-and-science-of-sleep-journal
The manuscript management system is completely online and includes a very quick and fair peer-review system, which is all easy to use. Visit http://www.dovepress.com/testimonials.php to read real quotes from published authors. 